\title{
Design of Multicode CDMA Systems for 3-D Stereoscopic Video over Wireless ATM Networks
}

\author{
Po-Rong Chang, Member, IEEE, Chin-Feng Lin, and M. J. Wu
}

\begin{abstract}
This paper investigates the application of multicode spread-spectrum code-division multiple-access (SS-CDMA) techniques to three-dimensional (3-D) stereoscopic video transmission over wireless asynchronous transfer mode (ATM) networks. Three-dimensional visual communications, made through the use of stereoscopic images, are able to achieve total display realism. Such services allow users to share the virtual reality (VR) world without any geographical restrictions. In order to create a 3-D system with two images (left and right) that should be transmitted over a bandlimited mobile channel simultaneously, a cost-effective Motion Picture Experts Group (MPEG)-based wavelet multiresolution coding with a joint motion and disparity compensation is developed to reduce a large amount of information contained in the images to meet the low-transmission rate limitation of mobile channels. However, the rapidly variable bit rate (VBR) characteristics of the MPEG-based 3-D videos seems a weakness to the transmission of such videos via a constant bit-rate (CBR) mobile channel. The ATM technique is especially well suited for VBR MPEG-based 3-D video because of its ability to allocate bandwidth on demand to these services. However, since the mobile radio has a limited channel capacity, the overall capacity of the traditional ATM-based SS-CDMA system may not be sufficient to accommodate the MPEG-based 3-D video services requested by the multiple mobile users simultaneously. To tackle this difficulty, a multicode CDMA technique is proposed to provide VBR MPEG-based 3-D video services by varying the number of spreading codes assigned to the 3-D video to meet its dynamic throughput requirement. Powerful forward error correction (FEC) codes are necessary to protect the video data so that it can be successfully transmitted at an acceptable signal power level. Two separate FEC code schemes are applied to the header and payload of an ATM cell containing 3-D video data, respectively. The ATM cell header is protected by a relatively powerful FEC code to ensure correct delivery and low cell loss rate (CLR). On the other hand, the ATM cell payload is encoded for varying degrees of error protection according to the priority of the payload data in 3-D videos. An adaptive FEC code combining scheme is proposed to provide the good protection for payload data with the maximization of its code rate to minimize the extra bandwidth for FEC overhead.
\end{abstract}

Index Terms-Multicode CDMA, 3-D video, wireless ATM.

\section{INTRODUCTION}

$\mathbf{I}$ $\mathrm{N}$ THE NEAR future, third-generation (3-G) mobile communications based on the asynchronous transfer mode (ATM) are developed to support a wide variety of multimeda services with diverse statistical characteristics and quality of service (QOS) requirements at cell and call levels. Among

Manuscript received August 5, 1997; revised May 5, 1999. This work was supported by the National Science Council, Taiwan, R.O.C., under Contract NCS 88-2213-E009-128.

The authors are with the Department of Communication Engineering, National Chiao-Tung University, Hsin-Chu, Taiwan, R.O.C.

Publisher Item Identifier S 0018-9545(00)02551-2. the various kinds of services, video service is becoming an important component of multimedia communication. In this paper, we are particularly interested in three-dimensional (3-D) stereoscopic video. One could acquire the 3-D video by wearing a portable stereoscopic eyeglasses with wireless communication capability. Stereoscopic image display is a simple and compact means of portraying depth information on a two-dimensional (2-D) screen. The binocular parallax or disparity between two images of the same scene, shot from two nearby points of view, contains information about the relative depths of the objects in the scene. Two pictures acquired in this manner form a stereopair. This relative depth can be deduced by humans, when each eye is presented with its corresponding image. In other words, the sense of stereovision can be simulated by acquiring these two pictures of the same scene with the disparity and by presenting the left picture to the left eye and the right picture to the right eye. Thus, stereoscopic image transmission requires twice the conventional monocular transmission bandwidth. However, several schemes [1]-[4] have been developed to exploit the disparity relation to achieve compression ratios higher than that obtained by the independent compression of the two pictures.

In order to further increase the compression ratio, in this paper, we apply the mixed-resolution coding technique [1] to incorporate with the disparity-compensated stereoscopic image compression. The mixed-resolution coding is a perceptually justified technique that is achieved by presenting one eye with a low-resolution picture and the other eye with one high-resolution picture. Psychophysical experiments [1] have shown that a stereo image pair with one high-resolution image and one low-resolution image provides almost the same stereoscopic depth to that of a stereo image with two high-resolution images. By combining both the mixed-resolution coding and disparity-compensated techniques, one reference (left) high-resolution image sequence can be compressed by a Motion Picture Experts Group (MPEG)-based motion-compensated discrete consine transform (DCT) scheme independent of the other (right) image sequence. By performing low-resolution disparity-compensated technique, the disparity is able to predict the low-resolution right image sequence from the left image sequence at a lower resolution using the disparity relation. The low-resolution images are obtained using the well-known novel wavelet decomposition [5], [6]. The another advantage of the wavelet decomposition is that it is very suitable for image compression. After the wavelet decomposition, an image is divided in several layers with different importances. Subimages at different layers correspond to different resolutions and different frequency ranges, which 
match the frequency-selected properties of human visual system. The wavelet low-resolution subimage in the left image is encoded by a well-known motion-compensated DCT scheme called the MPEG phase 2 (MPEG2) compressed video [9] since its characteristics are quite similar to the original image. In addition, the other high-frequency wavelet detail subimages in the left images are encoded by a motion-compensated scheme with a multiresolution scalar quantizer, where each quantization level corresponds to a detail subimage at its corresponding resolution level. On the other hand, the right image can be reconstructed from the left image by using the wavelet-based disparity-compensated technique. Since the estimation of both the motion vector and disparity is the computational burden of the joint motion and disparity-compensated technique, we apply the variable block-size multiresolution block matching method [3] to reduce their computational complexity. Both the estimated motion vector and disparity are then differential pulse code modulation (DPCM) coded, and all quantities are entropy coded prior to the transmission.

The ATM technology utilizes the nature of the traffic to effectively allocate the network resources via statistical multiplexing. Recently, wireless ATM has become an important research topic over the last three years [10]-[12]. Such services allow users to share novel multimedia applications without any geographical restrictions. J. Zhang et al. [13] have investigated an application of wireless ATM techniques to the transmission of MPEG video. However, they did not consider the problem of transmitting high-variable bit-rate (VBR) MPEG-based 3-D video over a narrow-band wireless channel. This problem could be ameliorated via multicode code-division multiple-access (CDMA) transmission [8], [14]-[16]. In other words, high data transmission rates are achieved by allocating more than one spreading code to a single MPEG-based 3-D video in order to create more than one virtual channel for the 3-D video. The code number assigned to each 3-D video is proportional to the dynamic source bit rate of 3-D video. Therefore, a spreading code assignment mechanism has been proposed to assign an appropriate code number to each 3-D video in order to achieve the maximum resource utilization. Furthermore, in order to avoid the self-interference that a 3-D video employing multiple codes may incur, the multiple codes to/from one 3-D video should be made orthogonal. This particular spreading coding scheme is called the concatenated orthogonal/PN spreading code [15] which is capable of subdividing a high rate stream belonging to a 3-D into several parallel lower basic bit-rate streams without self-interference. In addition, the quality of received 3-D video may degrade severely when the ATM cell loss occurs due to the multipath fading, interference, and channel noise. In Section IV, an adaptive forward error control (FEC) code combining scheme has been proposed to eliminate the cell loss according to both the statistical behavior and the importance of data in 3-D video. The objective of code combining is to maximize its code rate and to provide good error protection with reasonable delay. Simulation results show that this FEC code combining scheme is able to protect the 3-D video data and achieve the good received picture quality without a large amount of extra FEC overhead data. Moreover, Zhang and Lee [13] have shown that the error concealment techniques are very effective in improving both objec-

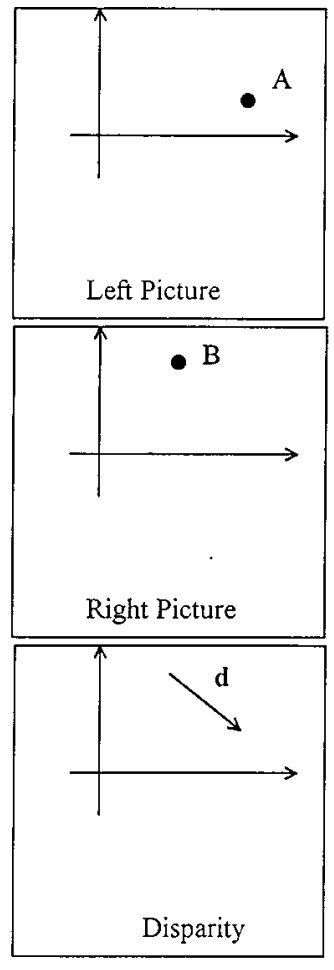

Fig. 1. Stereo disparity: A and B are matching points in the stereopair and d Id the disparity vector.

tive and subjective quality of received MPEG video via multipath fading channels. Therefore, in Section VI, a modification of both the spatial and temporal error concealment techniques [18]-[23], [35] is applied to recover the left picture in 3-D video with information loss. Moreover, a disparity error concealment technique is proposed to recover the damaged right image. Finally, a typical 3-D video test sequence is conducted to verify the effectiveness of the proposed ATM-based multicode CDMA system in various aspects.

\section{Stereoscopic IMAGE COMPRESSION USING MiXed-Resolution CODING TEChNiQues}

\section{A. Theory of Stereovision}

The sense of stereovision is normally simulated by viewing a true 3-D scene. It is possible to stimulate the sense of stereovision artificially by acquiring two pictures of the same scene from separated positions and by presenting the left picture to the left eye and the right picture to the right eye. Two pictures acquired in the manner form a stereopair. One of the most important ideas in the study of stereopairs is that of disparity. Fig. 1 illustrates the concept of disparity. Given a point A in the left picture, its matching point $\mathrm{B}$ in the right picture does not in general lie directly underneath $\mathrm{A}$. The vector connecting $\mathrm{B}$ to $\mathrm{A}$ has been called the disparity, the stereo disparity, the binocular disparity, and the binocular parallax of the point pair (A, B). The disparity $\mathbf{d}$ associated with the point pair (A, B) consists of two components: a horizontal component $d_{h}$ and a vertical component $d_{v}$. Depending on the camera geometry being used, each component of the disparity can be either positive or negative. When negative disparity occurs, the scene being viewed will 
appear to be floating in the space between the viewer's eyes and monitor. This type of imagery cannot be produced without the aid of stereoscopic devices such as shutter glasses. For another case of parallel axes camera geometry, the vertical component of disparity is always zero and the horizontal component of the disparity is always positive. This implies that the parallel axes geometry processes a simple mathematical relationship between the disparity of a point pair and the distance to the object it represents. In general, the disparity vector $\mathbf{d}$ can be used to predict one image of stereopair from the other. For example, given a luminance level of the left picture at a position $\mathrm{p}, I_{L}(\mathrm{p})$, the luminance level of its corresponding right picture can be calculated as

$$
I_{R}(\mathbf{p})=I_{L}(\mathbf{p}+\mathbf{d})
$$

where $\mathbf{d}$ denotes the disparity vector whose direction is from left to right.

\section{B. Mixed-Resolution Coding for Stereopair Data Compression Using Wavelet Multiresolution Techniques}

Mixed-resolution coding is a perceptually justified technique for compressing stereopairs. The compression is achieved by presenting one eye with a low-resolution picture and the other eye with a high-resolution picture. Psychophysical experiments [1] have shown that a stereo image with one high-resolution image and one lower resolution image is sufficient to provide almost the same stereoscopic depth as that of a stereo image with two high-resolution images. Thus, the eye/brain can easily fuse such stereopairs and perceive depth in them. In summary, the concept of mixed-resolution technique can be symbolically represented by the following:

$$
\begin{aligned}
(\text { Stereo image }) \approx & (\text { High-resolution left image }) \\
& +(\text { Low-resolution right image })
\end{aligned}
$$

$$
\begin{aligned}
(\mathrm{LR} \text { right image })= & (\text { LR left image })+(\text { Disparity between } \\
& \text { both LR images })
\end{aligned}
$$

where LR denotes the low-resolution image. From the above discussion, the mixed-resolution coding is able to significantly reduce the bit rate required to transmit a stereo image with two high-resolution images. To implement the mixed-resolution coding, one of the novel techniques is based on the well-known wavelet multiresolution signal representation [5], [6]. In the remainder of this section, we would give a brief review on the wavelet multiresolution technique.

Mallat [6] showed that the 2-D wavelet at a given resolution $2^{m}$ can be completely represented by three separable orthogonal 2-D wavelet basis functions in $\left.L^{2}\left(R^{2}\right): 1\right) \psi_{m n l}^{1}(x, y)=$ $\phi_{m n}(x) \psi_{m l}(y)$; 2) $\psi_{m n l}^{2}(x, y)=\psi_{m n}(x) \phi_{m l}(y)$; and 3) $\psi_{m n l}^{3}(x, y)=\psi_{m n}(x) \psi_{n l}(y)$, where $n$ and $l$ are shift parameters for $x$ and $y$ directions, respectively. The one-dimensional (1-D) wavelet is defined by

$$
\psi_{m, n}(x)=2^{-(m / 2)} \psi\left(2^{-m} x-n\right), \quad(m, n) \in Z^{2}
$$

where $\psi$ is the mother wavelet, $m$ is a scaling parameter and $n$ is a shift parameter, and the 1-D scaling function is defined by

$$
\phi_{m, n}(x)=2^{-(m / 2)} \phi\left(2^{-m} x-n\right)
$$

where $\phi$ is the mother scaling function. Hence, a 2-D wavelet transform of image $f(x, y)$ between the scale $2^{1}$ and $2^{M}$ can be represented as a sequence of subimages

$$
\left\{S_{2^{M}} f,\left[W_{2^{M}}^{j} f\right]_{j=1,2,3}, \cdots,\left[W_{2^{1}}^{j} f\right]_{j=1,2,3}\right\}
$$

where $S_{2^{M}} f$ is the approximation of image $f(x, y)$ at the lowest resolution $2^{M}$ and the detail subimages at resolution $2^{m}$ are defined in terms of an inner product

$$
\begin{gathered}
W_{2^{m}}^{j} f=\left(\left\langle f(x, y), \psi_{m n l}^{j}(x, y)\right\rangle\right)_{(n, l) \in Z^{2}} \\
1 \leq j \leq 3 .
\end{gathered}
$$

The 2-D separable wavelet decomposition can be implemented first in columns and then in rows independently by using the high-pass filter $g(n)=(-1)^{n} h(-n+1)$ and the low-pass filter $h(n)=2^{1 / 2} \int \phi(x-n) \phi(2 x) d x$, respectively. This decomposition provides subimages corresponding to different resolution levels and orientations with resolution depth $M$. Moreover, Zhang and Zafar [5] have shown that the decomposed image forms a pyramid structure up to $M$ layers with three detail subimages in each layer and one lowest resolution subimage on the top. The pyramid structure of the 2-D wavelet decomposition with resolution depth three consists of a total of seven subimages $\left\{S_{4}, W_{4}^{1}, W_{4}^{2}, W_{4}^{3}, W_{2}^{1}, W_{2}^{2}, W_{2}^{3}\right\}$. The resolution decreases by a factor of four (two in the horizontal direction and two in the vertical resolution) with each layer increased.

After the wavelet decomposition, an image is divided into several layers with different importances. Subimages at different layers correspond to different resolutions and different frequency ranges, which match the frequency-selected properties of the human visual system. It is well known that human viewers are more sensitive to lower frequency than higher frequency image components. Additionally, energies after wavelet decomposition become highly nonuniform. The higher the layer is, the higher the energy becomes. For example, over $80 \%$ of the energy is concentrated in the subimage $S_{4}$. For the implementation of mixed-resolution coding, the low-resolution right image can be obtained by performing the wavelet decomposition. However, in order to further achieve the higher compression ratio, the next section will present a new disparity-compensated multiresolution coding scheme which is able to compress the stereo image by aid of low-resolution disparity estimation.

\section{JoINT MOTION/DisPARITY-COMPENSATED WAVELET MultiRESOlution CODING FOR 3-D STEREO IMAGE SEQUENCE COMPRESSION}

This section presents a joint motion/disparity-compensated wavelet multiresolution coding for 3-D stereo image sequence to achieve the higher compression ratio. The left image sequence (reference image sequence) is compressed independent of the right image sequence using a combination of discrete 


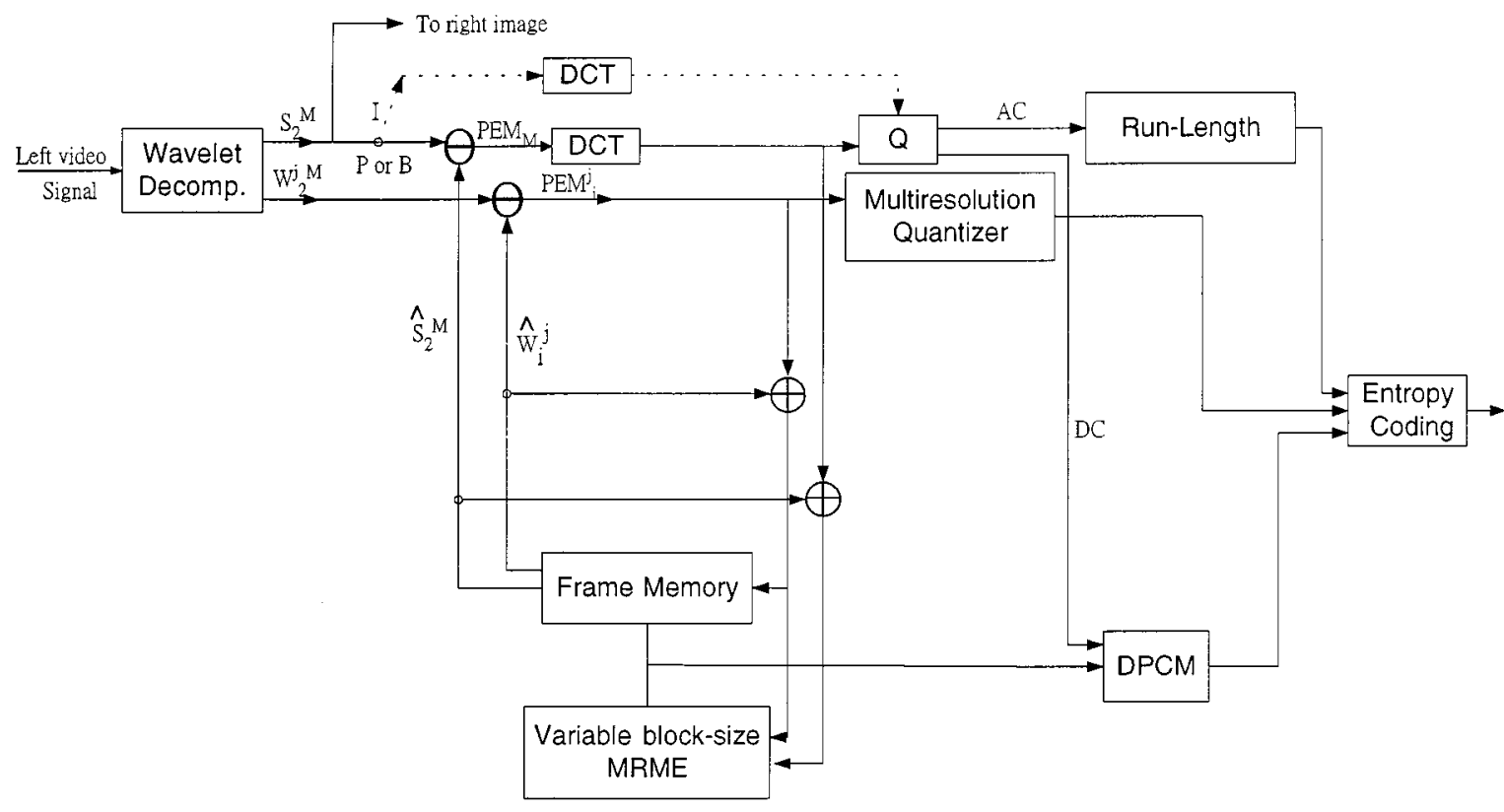

Fig. 2. Interframe MPEG-based hybrid motion-compensated DPCM/DWT/DCT scheme for left image sequence using a variable block-size multiresolution motion estimation (MRME).

wavelet transform (DWT) and MPEG-based motion-compensated DCT coding [5], [9]. Instead of transmitting the right image directly, a new interview hybrid DPCM/DWT with scalar quantization (SQ) is proposed to provide the coding information of both the disparity and the prediction error (residual) image to reconstruct the right image from the left image at the receiver. Moreover, by employing the mixed-resolution psychophysical experiments, one may perform the coding mechanism in the low-resolution manner, where the disparity is estimated from both the left and right images at a low-resolution level and the prediction error image is also obtained by the interview DPCM/DWT/SQ scheme from the low-resolution disparity-compensated right image.

\section{A. MPEG-Based Hybrid Motion-Compensated DPCM/DWT/DCT Coding Scheme for Left Image Sequence}

The left image stream is compressed using the hybrid DPCM/DWT/DCT scheme illustrated in Fig. 2 independent of the right image stream. Using wavelet decomposition, an orignal image $S$ is first decomposed into a subimage at the lowest resolution level $M, S_{M}$, and $3 \times M$ detail subimages $\left\{W_{2^{m}}^{j} ; M=1,2, \cdots M, j=1,2,3\right\}$. Since the statistics of the subimage at the lowest resolution are quite similar to the statistics of the original image, an MPEG-based motion-compensated DCT coding technique is used to encode it. Three main picture types are generated from the MPEG coding mechanism. Intrapictures (I-pictures) are coded using DCT transform without reference to other pictures. The I picture at the beginning of a group-of-pictures (GOP's) serves as a basic entry point to facilitate random seek and also provides the coding robustness to transmission error, but is coded with only moderate compression to reduce the spatial redundancies. Predictive $(\mathrm{P})$ pictures are coded more efficiently using motion-compensated prediction from past
I or $\mathrm{P}$ pictures and generally used as a reference for further prediction. Bidirectionally predictive (B) pictures provide the highest degree of compression, but require both past and future reference (I or P) pictures for motion compensation. For either the $\mathrm{P}$ or B frames, its prediction error image $\mathrm{PEM}_{M}$ is encoded using DCT transform and would then be used to obtain the motion-compensated subimage at the lowest resolution, i.e., $\hat{S}_{2^{M}}$. On the other hand, $3 \times M$ prediction error subimages $\left\{\mathrm{PEM}_{m}^{j} ; m=1,2, \cdots M, j=1,2,3\right\}$ are generated from their corresponding detail subimages $\left\{W_{2^{m}}^{j} ; m=1,2, \cdots, M\right.$, $j=1,2,3\}$, where each of them is encoded using a scalar quantizer with a quantization level corresponding to its resolution. Such a quantization scheme is called the multiresolution quantizer. These prediction error subimages are then applied for the construction of the motion-compensated detail subimages $\left\{\hat{W}_{2^{m}}^{j} ; m=1,2, \cdots, M, j=1,2,3\right\}$. Moreover, the motion vectors are DPCM coded, and a variable-length coding (VLC) is used for all the quantities including I frame and prediction error subimages. For color video frames, the motion vectors obtained from the luminance component of the color video frames are used as the motion vectors for both the $\mathrm{C}_{r}$ and $\mathrm{C}_{b}$ components because $Y$ component contains more than $60 \%$ of total energy in the original image. In this paper, the luminance component is decomposed into seven subimages. In the meantime, each chrominance component may be decomposed into four subimages since the size of either $\mathrm{C}_{b}$ or $\mathrm{C}_{r}$ component is $1 / 4$ of the original video frame size. The resulting subimages are illustrated in Fig. 3.

\section{B. Interview Disparity-Compensated Hybrid DPCM/DWT/SQ Scheme}

The aim of disparity estimation is the matching of corresponding picture elements in the simultaneous 2-D pictures of the same 3-D scene, viewed under different perspective angles. 


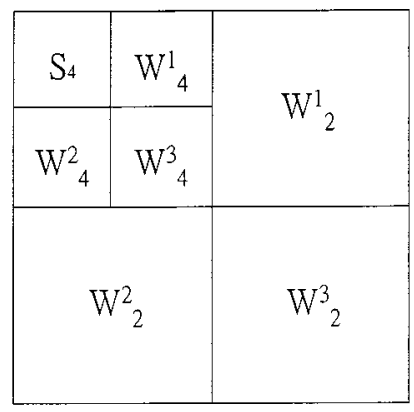

Y-component

\begin{tabular}{|c|c|}
\hline $\mathrm{S}_{2}$ & $\mathrm{~W}_{2}^{1}$ \\
\hline $\mathrm{W}_{2}^{2}$ & $\mathrm{~W}_{2}^{3}$ \\
\hline
\end{tabular}

Cb-component

\begin{tabular}{|c|c|}
\hline $\mathrm{S}_{2}$ & $\mathrm{~W}_{2}^{1}$ \\
\hline $\mathrm{W}^{2}$ & $\mathrm{~W}_{2}^{3}$ \\
\hline
\end{tabular}

Cb-component
Fig. 3. Wavelet decomposition of color image.

Two of those pictures may be the left and right views of a stereopair. A number of block-based disparity-compensated methods have been proposed for thecoding stereopairs [2]-[4]. With block methods, it is assumed that the disparity between the left image and right image is constant within a small 2-D block $B_{d}$ of $P_{d} \times Q_{d}$ pels (pixels). Therefore, the disparity d can be estimated by minimizing the $L_{1}$ norm of disparity prediction error such as

$$
\operatorname{DPE}(\mathbf{d})=\sum_{\mathbf{z} \in B_{d}}\left|I_{L}(\mathbf{z})-I_{R}(\mathbf{z}-\mathbf{d})\right| .
$$

Consider a block of $P_{d} \times Q_{d}$ pels centered around pel $\mathbf{z}_{0}$ in the left image (reference image). Assume that the maximum horizontal and vertical disparity displacements are $p_{d}$ pels and $q_{d}$ pels, respectively. Thus, the search region in the right image would be an area containing $\left(P_{d}+2 p_{d}\right)\left(Q_{d}+2 q_{d}\right)$ pels. A simplified version of the criterion of (8) is given by

$$
\begin{aligned}
\operatorname{DPE}\left(\mathbf{z}_{0}, x, y\right)= & \frac{1}{P_{d} Q_{d}} \sum_{|p| \leq P_{d} / 2} \sum_{|q| \leq Q_{d} / 2} \mid I_{L}\left(z_{1}+p, z_{2}+q\right) \\
& -I_{R}\left(z_{1}+p+x, z_{2}+q+y\right) \mid
\end{aligned}
$$

where $-p_{d} \leq x \leq p_{d},-q_{d} \leq y \leq q_{d}$, and $z_{1}$ and $z_{2}$ are the $x$ and $y$ coordinates of $\mathbf{z}_{0}$, respectively. The minimization of the disparity prediction error of (9) is performed using any one of the four promising methods used in motion estimation, i.e.: 1) full search; 2) 2-D logarithmic search; 3) three-step search; and 4) modified conjugate direction. According to the mixed-resolution psychophysical experiments, the block-based disparity estimation is performed at the low resolution. The computational complexity of the low-resolution disparity estimation becomes smaller due to the smaller search area at the lowest resolution. At the receiver, the low-resolution right subimage is estimated using the disparity from the low-resolution left subimage. A full-size reconstruction is obtained by upsampling a factor of four and reconstructing with the synthesis low-pass filter. However, in order to further improve the reconstruction quality of low-resolution right image, disparity has to be transmitted, together with the reconstruction error. In this section, we present an interview disparity-compensated hybrid DPCM/DWT/SQ scheme (shown in Fig. 4) to both improve the reconstruction image quality and achieve the higher compression ratio.

An original right image $S$ is first decomposed into a number of subimages $\left\{S_{2^{M}}, W_{2^{m}}^{j} ; m=1, \cdots M, j=1,2,3\right\}$. Only the lowest resolution subimage $S_{2^{M}}$ is considered in our system. After using the block-based disparity compensation scheme, the disparity-compensated right subimage $\hat{S}_{2^{M}, D}$ is estimated from its corresponding low-resolution left images using the low-resolution disparity. Then the lowest resolution prediction error subimages $\mathrm{PED}_{M}$ for the disparity-compensated subimage $\hat{S}_{2^{M}, D}$ is formed using the disparity compensation. Since all the pixels in $\mathrm{PED}_{M}$ are uncorrelated, it is suggested that the lowest resolution reconstruction error subimage $\mathrm{PED}_{M}$ is coded using an optimal Lloyd Max scalar quantizer (SQ) instead of DPCM. Finally, disparity vectors are DPCM coded, and all the quantities are entropy coded prior to the transmission.

\section{Multiresolution Estimation for Motion/Disparity Vectors}

Stereo image sequence processing requires the estimation of the displacements created by the motion of objects and also by the disparity between two views of the 3-D scene projected on the two images. The estimation of both the motion and disparity displacements may be performed separately. The motion estimation problem is similar to that of disparity estimation. The motion vector can be found by applying any one of the promising search methods to the minimization of motion prediction error. In order to further reduce both the computational complexity and searching time of above four methods, an efficient hierarchical block matching algorithm [3], [5] has been applied to both motion and disparity estimation, in which agreement of a large block is first attained and block size is subsequently and progressively decreased. One approach to hierarchical block matching uses multiple resolution versions of the image and variable block size at each level of the pyramid [3], [5]. In a multiresolution motion estimation (MRME) scheme, the motion vector field is first calculated for the lowest resolution subimage, which sits on the top of pyramid [3], [5]. Motion vectors at the lower layers of the pyramid are refined using the motion information obtained at higher layers and again propagated to the next pyramid level until the high-resolution level is reached. The motivation for using the MRME approach is the inherent structure of the wavelet representation. MRME schemes significantly reduce the searching and matching time and provide a smooth motion vector field. Moreover, it is well known that human vision is more perceptible to errors in low frequencies than those incurred in higher bands to be selective in spatial orientation and position, e.g., errors in smooth areas are more disturbing to a viewer than those near edges. The subimage $S_{4}$ contains a large percent of the total energy though it is only $1 / 16$ th of the original video frame size. Additionally, errors in higher layer subimages will be propagated and expanded to all the subsequent lower layer subimages. To tackle this difficulty, Zhang and Zafar [5] have proposed a variable block-size MRME scheme to take all these factors into considerations.

Similarly, Tzovaras et al. [3] have shown that the above variable block-size multiresolution block matching techniques are also valid for disparity estimation to reduce the amount of 


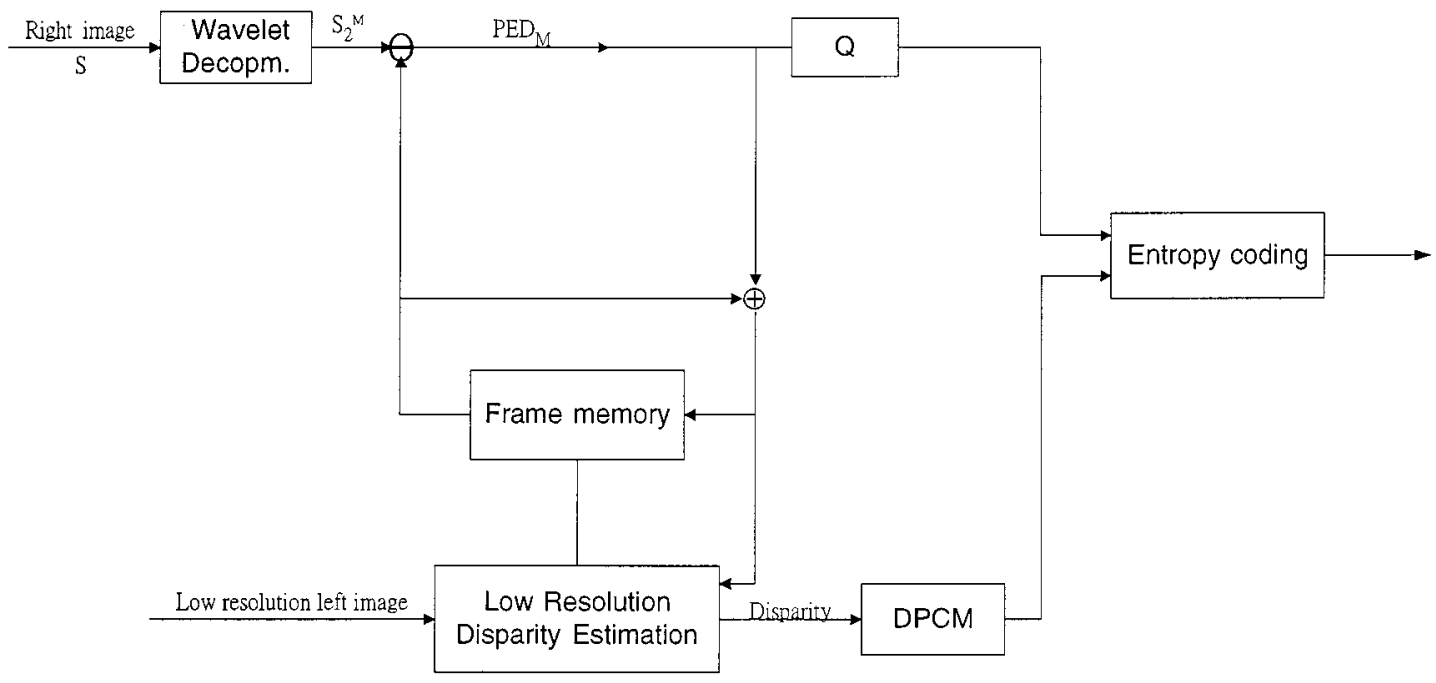

Fig. 4. Interview disparity-compensated hybrid DPCM/DWT/SQ scheme.

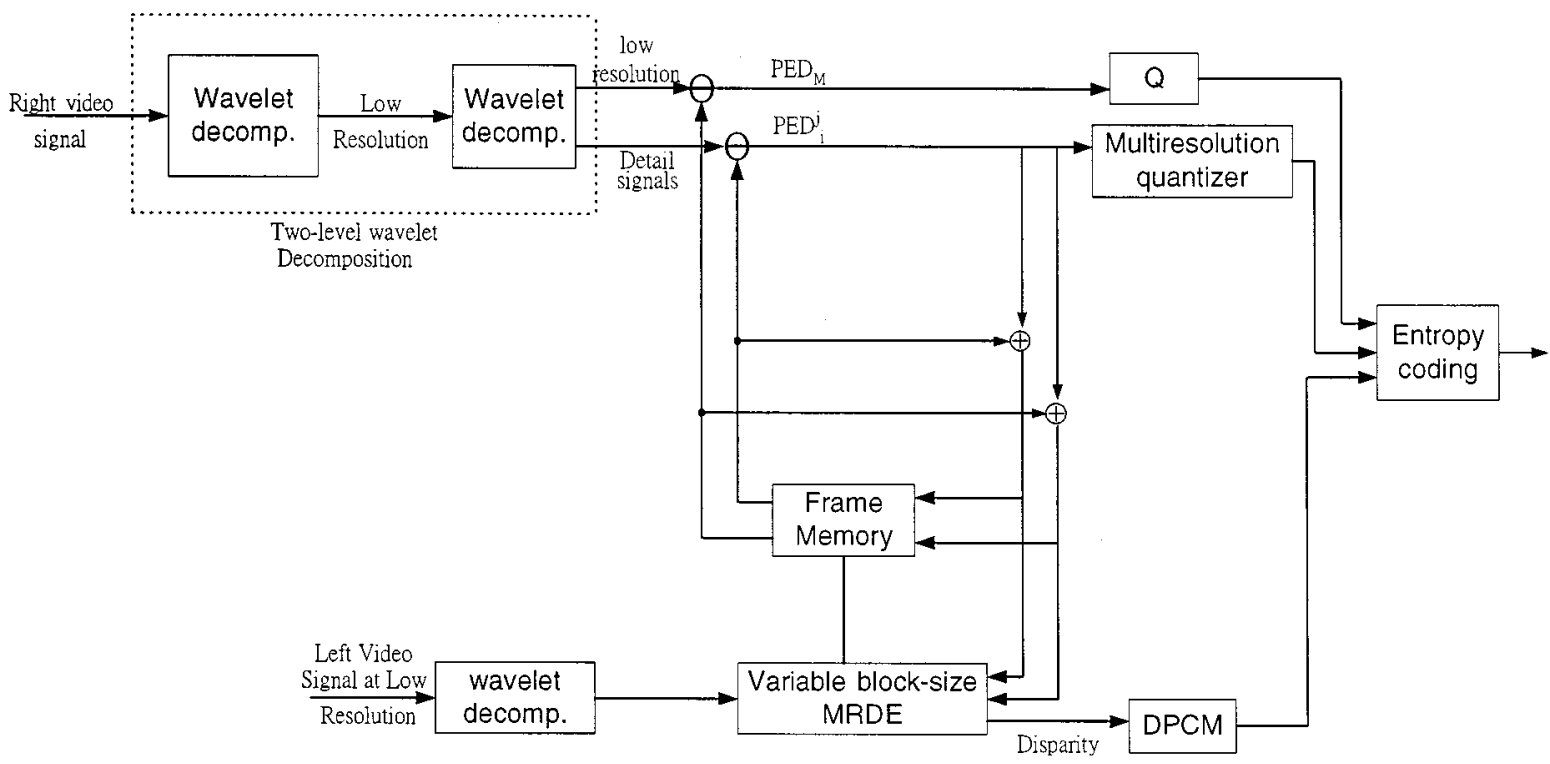

Fig. 5. Interview disparity-compensated DPCM/DWT/SQ scheme for low-resolution right and left image sequences using MRDE.

processing time. This particular disparity estimation scheme is called the variable block-size multiresolution disparity estimation (MRDE).

According to the mixed-resolution psychophysical experiments and the above concept, this paper presents a low-resolution MRDE scheme for disparity estimation and compensation to improve the computational efficiency of estimation. The low-resolution MRDE scheme requires a two-level wavelet decomposition. The first-level wavelet decomposition is used to obtain both the lowest resolution left and right video frames. The second-level wavelet decomposition is conducted to perform the MRDE at the lowest resolution. However, the quality of the reconstructed stereo image sequence may be degraded using only the motion and disparity vectors for some cases. In order to further improve the reconstruction image quality, both the motion and disparity vectors have to be transmitted together with prediction errors.

\section{Interview Disparity-Compensated Hybrid DPCM/DWT/SQ Scheme for Low-Resolution Right Image Sequence Using a Variable Block-Size MRDE Technique}

All the low-resolution right video frames can be estimated from their corresponding left video frames using the low-resolution variable block-size MRDE procedure described above. In order to improve the image quality of the reconstructed right video frames, both the disparity and reconstruction (prediction) errors should be transmitted. We use the architecture which is similar to interview hybrid DPCM/DWT/SQ scheme proposed in Section II-B to implement this concept. Fig. 5 shows its basic structure. The main difference between Figs. 4 and 5 is that Fig. 5 contains a two-level wavelet decomposition and a variable block-size multiresolution disparity estimation. Both the lowest resolution left and right video frames are obtained by the firstlevel wavelet decomposition. The resulting low-resolution left 


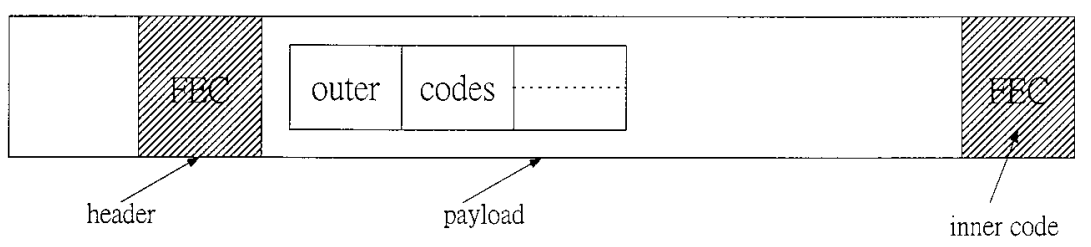

Fig. 6. Logical structure of a wireless ATM cell outer/inner FEC code combining.

and right video frames are again decomposed into the secondlevel lowest resolution subimage and the wavelets in different scales and resolutions. The second-level prediction error subimages $\left\{\mathrm{PED}_{M}, \mathrm{PED}_{m}^{j} ; m=1, \cdots M, j=1,2,3\right\}$ for low-resolution disparity-compensated subimages are formed using the low-resolution variable block-size MRDE scheme. Like the disparity-compensated interview compression scheme, the $\mathrm{PED}_{M}$ is coded using an optimum scalar quantizer, and the subimages $\mathrm{PED}_{m}^{j}$ are coded using multiresolution quantizer. For color stereo video frames, the disparity vectors obtained from their luminance components are used as the disparity vectors for their $C_{r}$ and $C_{b}$ chrominance components.

\section{DESIGN OF WIRELESS ATM CELLS FOR MPEG-BASED Multiresolution 3-D Stereo VideOs}

This section presents a design philosophy of wireless ATM cells for 3-D stereo videos based on the concept of ATM cell design for MPEG videos. Zhang et al. [13] have proposed a wireless ATM cell model for MPEG-2 videos which is quite similar to Lei's cell structure [22] with a header of 5 bytes and a payload of 48 bytes for MPEG-2 over wireline ATM. However, wireless multipath fading, interference, and noise tend to cause significant ATM cell loss. A single cell loss will result in the severe MPEG image quality degradaion when the cell carries a large amount of payload data. Liu and El Zarki [29] have suggested that the wireless ATM cell should not carry too much data to avoid the loss of large amount of image data in a single cell loss. Furthermore, FEC codes are necessary to protect either the ATM cell header or payload data so that it can be successfully transmitted at an acceptable signal power level to provide adequate image quality. However, the extra bandwidth for FEC overhead for wireless ATM cell is also critical in the narrow-band wireless networks. This section will investigate the appropriate tradeoff between forward error control and MPEG-based multiresolution 3-D video transmission quality.

Two separate error control coding schemes are applied to the header and payload of wireless ATM cell illustrated in Fig. 6, respectively. Usually, an ATM cell consists of a 5-byte header. Raychaudhur [8] showed that the ATM header over a wireless nework could be compressed to 2 or 3 bytes. For simplicity, a 2-byte compressed header will be used in the wireless ATM cell. A shortened BCH code of $(31,16,3)$ is suggested to protect this header. On the other hand, a forward error control coding scheme with unequal error protection (UEP) is applied to the payload according to the priority for its data types in 3-D video with a wavelet multiresolution coding. The allocation of priority levels can be performed in a hierarchical multiresolution manner to provide scalable video at different resolutions. There are four priority levels for the $Y$ component in the wavelet multiresolution coding with a seven-subimage decomposition and three priority levels for either $\mathrm{C}_{b}$ or $\mathrm{C}_{r}$ component in the wavelet coding with a four-subimage decomposition. For the $Y$ component, the encoded bits from the low-resolution subimage $S_{4}$ could be allocated the highest priority level (priority level 1), three detail subimages $W_{4}^{1}, W_{4}^{2}$, and $W_{4}^{3}$ allocated the second highest priority level (priority level 2), two detail subimages $W_{2}^{1}$ and $W_{2}^{2}$ allocated the third highest priority level (priority level 3), and a detail subimage $W_{2}^{3}$ allocated the lowest priority level (priority level 4). Since the lowest priority detail subimage $W_{2}^{3}$ is visually less important than the other subimages and has very little impact on video quality, it is not considered in the transmission via the wireless channel. Thus, there are $\mathrm{BCH}$ codes with three different error protection levels of (255 215), (255 223), and (255231) which are used to protect the highest priority subimage $\left(S_{4}\right)$, the second highest priority subimages $\left(W_{4}^{1}, W_{4}^{2}, W_{4}^{3}\right)$, and the third highest priority subimages $\left(W_{2}^{1}, W_{2}^{2}\right)$, respectively. For either the $\mathrm{C}_{b}$ or $\mathrm{C}_{r}$ components, two BCH codes of (255 215) and (255223) are applied for the protection of the highest priority subimage $S_{2}$ and the second highest priority subimages $\left(W_{2}^{1}, W_{2}^{2}\right)$, respectively. Its lowest priority subimage $W_{2}^{3}$ is not considered in the transmission. From the above discussion, the prioritization of the wavelet multiresolution coding for 3 -D video is actually performed at the spatial domain. On the other hand, the prioritization for the multiresoulation 3-D video using the MPEG coding mechanism should be also considered in the temporal domain. Especially, there are three priority levels involved in the low-resolution subimage $S_{4}$ at three different time points in the temporal line since $S_{4}$ is encoded using the MPEG coding scheme. For the MPEG bit stream generated from the the low-resolution subimage $S_{4}$, I frame information tends to be the most important data for transmission because the effect of losses on this frame will tend to propagate until the next I frame and then cause no temporal prediction for $\mathrm{P}$ and $\mathrm{B}$ frames. The $\mathrm{P}$ and $\mathrm{B}$ frames are, respectively, the medium and the least important data for transmission since $\mathrm{P}$ frame is usually treated as a reference for the temporal prediction of $\mathrm{B}$ frames. Therefore, three $\mathrm{BCH}$ codes with three different error protection levels, i.e., of (255215), (255 223), and (255 231) are used to protect I, P, and B frames at three different time points in the temporal line, respectively. The above joint MPEG source coding and channel coding is also valid for either the $\mathrm{C}_{b}$ or $\mathrm{C}_{r}$ components. Next, considering the second highest priority detail subimages in the spatial domain, the essential components in the multiresolution motion-compensated coding scheme become the prediction error subimages $\mathrm{PEM}_{4}^{1}, \mathrm{PEM}_{4}^{2}$, and $\mathrm{PEM}_{4}^{3}$ together with their associated motion vectors for the $Y$ component, and $\mathrm{PEM}_{2}^{1}$ and $\mathrm{PEM}_{2}^{2}$ 
together with their associated motion vectors for either the $C_{b}$ or $\mathrm{C}_{r}$ components. It is found that there is no priority difference between any two prediction error subimages located at two different time points in the temporal line. In other words, the priority for each prediction error subimage is only considered in the spatial domain. Thus, $\mathrm{BCH}(255223)$ is used to protect those prediction error subimages with the second highest priority (in the spatial domain). Finally, $\mathrm{BCH}(255231)$ is used to protect the lowest priority prediction error subimages, i.e., $\mathrm{PEM}_{2}^{1}$ and $\mathrm{PEM}_{2}^{2}$ for $Y$ component. However, the lowest priority prediction error subimages for both $\mathrm{C}_{b}$ and $\mathrm{C}_{r}$ components are not considered in the transmission via the wireless channel. Note that the motion-compensated mechanism for the detail subimages is quite similar to the generation of $\mathrm{P}$ frames in the MPEG coding. However, it did not include the DCT transform.

As a result, the degree of error protection for the low-resolution subimage $S_{4}$ should be considered in both the spatial and temporal domains whereas the prediction error subimages are only performed in the spatial domain. Therefore, $\mathrm{BCH}(255215)$ is used to protect the I frame in $S_{4}$ which is the highest priority subimage in both the spatial and temporal domains. However, the P frame in $S_{4}$ is the highest priority subimage in the spatial domain, but the second highest priority one in the temporal domain. Thus, it is protected by $\mathrm{BCH}(255223)$. Similarly, the third highest priority subimage in the temporal domain which is the $\mathrm{B}$ frame in $S_{4}$ could be protected by $\mathrm{BCH}(255231)$. On the other hand, the same error protection scheme can be applied to the detail subimages in both the spatial and temporal domains. Fig. 7 shows the basic concept of the priority in both the spatial and temporal domains for the MPEG-based multiresolution 3-D video including the I, P, and B frames generated from the low-resolution subimage (along the temporal line and fixed at the highest priority point in the spatial line) and detail subimages (along the spatial line and fixed at the highest priority point in the temporal line). Note that, for abbreviation, the I, P, and B frames generated from the low-resolution subimage $S_{4}$ are called the low-resolution I, P, and $B$ frames, respectively. In addition, it is assumed that the identical error protection is applied to any two different picture frames when they have the same priority level in either spatial or temporal domain. For example, the second highest priority low-resolution $\mathrm{P}$ frame in the temporal domain and the second highest priority detail subimages in the spatial domain are protected by the same FEC code BCH(255 223). Note that the above-mentioned priority analysis is considered in the frame level. In the meantime, it is desirable to consider the priority analysis for the data within each picture frame.

By analyzing the data types in each of I, P, and B frames, it is found that there are eight priority levels for I frame and nine priority levels for either the $\mathrm{P}$ or $\mathrm{B}$ frames. For the sake of simplifying the description and giving a more compact definition for the picture frame in MPEG-based multiresolution 3-D stereo video, it is suggested that each frame may include the disparity information. This particular frame generated from both the left and right low-resolution subimages is called either the low-resolution enhanced I, P, or B frame at its corresponding time instant. Thus, a single low-resolution enhanced frame is able to replace two picture frames generated from both the left and right low-resolution subimages via the multiresolution disparity-com- pensated coding scheme. Moreover, the prediction error signals for the disparity-compensated subimages should be considered in the prioritization. For example, the disparity-compensated prediction error $\mathrm{PED}_{4}$ together with the disparity is able to reconstruct the low-resolution subimage in the right image from the low-resolution subimage $S_{4}$ in the left image. Table I-(A) illustrates the priority for each data type in either the low-resolution enhanced I, P, or B frame with disparity information generated from the low-resolution subimages in MPEG-based multiresolution 3-D stereo video. For low-resolution enhanced I frames, the highest priority is given to the frame header, the second highest priority is given to the slice header, the third highest priority is given to macroblock (MB) header, the fourth highest priority is given to dc DCT coefficients, the fifth highest priority is given to disparity vectors, and the lowest priority is given to high-frequency ac DCT coefficients. Correct reception of data types at priority levels 1-4 would guarantee the quality of a low-resolution left image sequence. The quality of right image sequence is maintained at an acceptable level when both the disparity vectors at priority level 5 and the data types at priority levels 1-4 have been correctly received. Certainly, correct reception of all the data types would provide the highest 3-D video resolution. For either low-resolution enhanced P or B frames, the fourth, fifth, and six th highest priority levels are assigned to the motion vectors, disparity vectors, and dc DCT coefficients, respectively, since the availability of partial motion/disparity vector information may be sufficient to produce reasonable low-resolution left and right images, even in the event of absence of DCT coefficient information due to channel losses. Moreover, Table I-(B) shows the priority for each data type in both the second and third highest priority detail subimages with disparity information. Moreover, considering an extension to the above low-resolution enhanced frame, a new abbreviated name called the 3-D enhanced frame containing the low-resolution enhanced frame, disparity information, both the second and third highest priority detail subimages, and the lowest priority detail subimages is proposed to replace in both the left and right image frames belonging to the traditional 3-D video via the MPEG-based joint motion/disparity wavelet multiresolution coding scheme. It yields a complete compact description for the MPEG-based multiresolution 3-D video. From the above discussion, the prioritization in MPEG-based multiresolution 3-D video seems quite difficult because it includes three priority categories, i.e., priority for each 3-D enhanced picture frame in both the spatial/temporal domains and the importance of data types belonging to the frame. Actually, the disparity domain (the fourth priority category) should be considered in the 3-D enhanced frame. For the sake of simplifying the priority analysis, the third data priority category may include the disparity information via the proposed multiresolution disparity-compensated coding scheme. This would eliminate the complicated priority analysis in the disparity domain. A concatenated FEC coding scheme is proposed to overcome this difficulty. The "inner" FEC code is used to protect each frame according to its priority in both the spatial and temporal domains. Undoubtedly, the above-mentioned $\mathrm{BCH}$ code for each picture frame is chosen as the best candidate for the inner code. On the other hand, an outer FEC code is proposed to protect the high-priority data within each frame twice in accordance with the importance of their data type. 


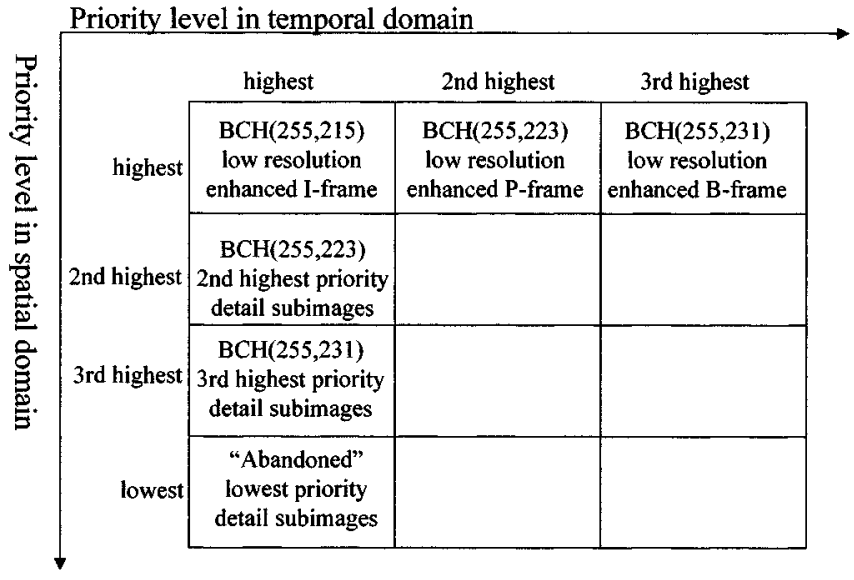

Fig. 7. Inner BCH-coded unequal error protection (UEP) scheme for each MPEG-based multiresolution 3-D enhanced picture frame according to its priority in both the spatial and temporal domains. Note that each of the low-resolution enhanced I, P, and B frames, and the detail subimages with three different priority levels includes the disparity information.

Assume that the frame header is included in a RM (resource management) cell with a high-error protection which is used to provide the current frame information and its resource management status for mobile receivers before transmitting the ATM cells. Hence, the outer FEC codes are proposed to protect the second, third, fourth, and fifth highest priority data (slice header, MB header, dc values, and disparity) for the low-resolution enhanced I frames and to protect the second, third, fourth, fifth, and sixth highest priority data (slice header, MB header, motion vectors, disparity, and dc values) for either the low-resolution enhanced P or B frames. Without the outer FEC protection, the remaining low-priority data are still protected by the inner codes. For the sake of reducing the implementation complexity, a class of shortened $\mathrm{BCH}$ codes is chosen for the design of the outer FEC coding. In the near future, we will concatenate either the inner rate compatible punctured convolutional (RCPC) code [25] or turbo code [36] with the proposed outer $\mathrm{BCH}$ code to further reduce the BER floor. Note that the adaptive outer error code combining is also suitable for the second and third highest priority detail subimages with disparity information according to the priority rank illustrated in Table I-(B). Their second, third, fourth, and fifth highest priority data (slice header, macroblock header, motion vector, and disparity) are protected by the same outer FEC coding scheme. The next section will discuss more details of the code selection principle for outer codes. In summary, Fig. 7 highlights the inner BCH-coded UEP scheme for each 3-D enhanced picture frame according to its priority in both the spatial and temporal domains. Note that the lowest priority detail subimages are not considered in the UEP scheme. On the other hand, Table I shows the priority bank for the data types belonging to each 3-D enhanced picture frame in accordance with the outer BCH-coded UEP scheme.

\section{A. Code Selection Principle for Outer FEC Codes According to Data Partitioning}

The work by Parthasarthy et al. [26] which describes a code selection strategy in the case of a normal RS code scheme for video traffic over wireline ATM networks is the closest to this
TABLE I

PRIORITY RANK FOR THE DIFFERENT DATA TyPeS Within EACH 3-D ENHANCED FRAME IN MPEG-BASED WAVELET MultiResolution 3-D VIDEO

(A) Priority rank list for the enhanced I-, P-, and B-frames generated from the low resolution subimages with disparity information

\begin{tabular}{ll}
\hline enhanced I-frames & 1. Frame header \\
2. Slice headers \\
3. Macroblock headers \\
4. DC coefficients \\
5. Disparity vectors \\
6. Low frequency AC coefficients \\
7. Disparity-compensated prediction error subimages \\
8. High frequency AC coefficients
\end{tabular}

enhanced P- and B-frames 1. Frame header

2. Slice headers

3. Macroblock headers

4. Motion vectors

5. Disparity vectors

6. DC coefficients

7. Low frequency $\mathrm{AC}$ coefficients

8. Disparity-compensated prediction error subimages 9. High frequency $\mathrm{AC}$ coefficients

(B) Priority rank list for the 2nd (or 3rd) highest priority detail subimages with disparity information

1. Frame header
2. Slice header
3. Macroblock header
4. Motion vectors for detail subimages at priority 2 (or priority 3 )
5. Disparity vectors for detail subimages at priority 2 (or priority 3 )
6. Motion-compensated prediction error subimages at priority 2 (or priority 3 )
7. Disparity-compensated prediction error subimages at priority 2 (or priority 3 )

paper. However, in [26], the MPEG video protected by the use of concatenated code according to its priority has not been considered. The objective of code selection is to minimize the outer code overhead and to provide good protection with reasonable delay. In other words, we choose an appropriate class of outer codes which maximizes the average code rate (for a coded ATM cell) given by

$$
\bar{R}_{c}=\frac{n_{m}^{c}}{\left(n_{h}^{c}+n_{p}^{c}\right)}
$$

where $n_{m}^{c}$ denotes the average number of message bits in a coded ATM cell and $n_{h}^{c}$ and $c_{p}$ represent the average numbers of bits in a coded header with $\mathrm{BCH}(31,16)$ and in a coded payload with inner/outer FEC coding, respectively. The maximization of $\bar{R}_{c}$ is over all the shortened BCH codes. Unfortunately, it is hard to characterize $\bar{R}_{c}$ directly in accordance with the variable bit rate (VBR) characteristics of the high-priority data belonging to each 3-D enhanced frame in MPEG-based multiresolution 3-D video. To overcome this difficulty, evaluate the average code rate for each of three essential components in the 3-D enhanced frame, i.e., low-resolution enhanced frame, the second 
and third highest priority detail subimages with disparity information, separately. Moreover, for the sake of simplifying the evaluation of the average code rate, the expression of (10) can be approximated by

$$
\bar{R}_{c} \approx \frac{\overline{\text { Slice }}}{\overline{C \text { Slice }}+\overline{\text { Code }}_{i n}+\overline{C A T M ~} h}
$$

where $\overline{\text { Slice }}$ is the average number of message bits in a slice and $\overline{C \text { Slice }}$ denotes the average number of bits in a coded slice using outer coding scheme only. $\overline{\operatorname{Code}}_{i n}$ and $\overline{C A T M} h$ represent the average numbers of bits in the inner code overheads and in the coded ATM headers within a slice, respectively. Let $m$ denote the number of message bits in the inner code. In other words, each ATM cell with inner coding can carry $m$ message bits per cell. Note that the coded bits generated by outer coding are treated as the message bits for the inner coding. Hence, the average number of ATM cells for a slice is $\overline{\lceil\text { CSlice }} / m\rceil\left(=\bar{n}_{\text {cell }}\right)$, where $\lceil x\rceil$ denotes the smallest integer which is greater than $x$. First, the low-resolution enhanced I, $\mathrm{P}$, and B frames with disparity information generated from the low-resolution subimages in both the left and right image sequences are regarded as a typical example to illustrate the evaluation of the average code rate $\bar{R}_{c}$. For the low-resolution enhanced I, P, and B frames, it is found that $\overline{\operatorname{Code}}_{i n}=(255-m) \times \bar{n}_{\text {cell }}$ and $\overline{C A T M ~} h=$ $31 \times \bar{n}_{\text {cell }}$, where $m=215$ (I frame) or 223 (P frame) or 231 (B frame). Therefore, the expression of (11) becomes

$$
\bar{R}_{c} \approx \frac{\overline{\text { Slice }}}{\left\{\overline{C \text { Slice }}+[(255-m)+31] \times\left\lceil\overline{\left.\left.\frac{C \text { Slice }}{m}\right\rceil\right\}}\right.\right.}
$$

where $m=215$ or 223 or 231 . Note that (12) is also valid for the second and third highest priority detail subimages with disparity information by employing $m=223(\mathrm{BCH}(255223))$ and $m=231(\mathrm{BCH}(255213))$, respectively.

Since $\overline{\text { Slice }}$ is a fixed value independent of outer coding scheme, the maximization of $\bar{R}_{c}$ then becomes the minimization of $\overline{C \text { Slice }}$. The expression of $\overline{C \text { Slice }}$ for either the low-resolution enhanced I, P, or B frames with disparity information generated from low-resolution subimages in 3-D video is given by

$$
\begin{aligned}
\overline{C \text { Slice }}= & \overline{C \text { Slice } h}+\overline{C M B h} \times \bar{n}_{M B}+\overline{C D C}_{Y} \\
& \times \bar{n}_{Y}+\overline{C D C}_{U} \times \bar{n}_{U}+\overline{C D C}_{V} \times \bar{n}_{V} \\
& +\overline{C D I S} \times \bar{n}_{D I S}+\overline{A C}+\overline{P E D}_{4}(Y) \\
& +\overline{P E D}_{2}\left(C_{b}\right)+\overline{P E D}_{2}\left(C_{r}\right)
\end{aligned}
$$$$
\text { for I frames }
$$

or

$$
\begin{aligned}
\overline{C \text { Slice }}= & \overline{C \text { Slice } h}+\overline{C M B h} \times \bar{n}_{M B}+\overline{C M V} \\
& \times \bar{n}_{m v}+\overline{C D I S} \times \bar{n}_{D I S}+\overline{C D C}_{Y} \times \bar{n}_{Y} \\
& +\overline{C D C}_{U} \times \bar{n}_{U}+\overline{C D C}_{V} \times \bar{n}_{V}+\overline{A C} \\
& \cdot \overline{P E D}_{4}(Y)+\overline{P E D}_{2}\left(C_{b}\right)+\overline{P E D}_{2}\left(C_{r}\right) \\
& \text { for P or B frames }
\end{aligned}
$$

where $\overline{C \text { Slice } h}$ is the average number of bits in a coded slice header using outer FEC coding. $\overline{C M B h}$ denotes the average number of bits in a coded MB header and $\bar{n}_{M B}$ is the average number of MB's within a slice. $\overline{C D C}_{X}$ denotes the average number of bits in the coded dc values in $X$ block and $\bar{n}_{X}$ represents the average number of $X$ blocks in a slice, where $X=Y$ or $\mathrm{U}\left(C_{b}\right)$ or $\mathrm{V}\left(C_{r}\right) . \overline{C M V}$ is the average number of bits in a coded motion vector and $\bar{n}_{m v}$ is the average number of motion vectors in a slice. $\overline{C D I S}$ presents the average number of bits in a coded disparity vector and $\bar{n}_{D I S}$ is the average number of disparity vectors in a slice. Note that the above parameters are encoded using outer FEC coding scheme only. Meanwhile, $\overline{A C}$ denotes the average number of bits in low-priority ac DCT coefficients within a slice without outer FEC coding. $\overline{P E D}_{4}(Y)$, $\overline{P E D}_{2}\left(C_{b}\right)$, and $\overline{P E D}_{2}\left(C_{r}\right)$ represent the average numbers of bits in the disparity-compensated prediction errors for the $Y, C_{b}$, and $C_{r}$ components of the low-resolution subimage in the right image without outer coding, respectively.

For the second highest priority detail subimages with disparity information, there are three prediction error subimages $\mathrm{PEM}_{4}^{1}, \mathrm{PEM}_{4}^{2}$, and $\mathrm{PEM}_{4}^{3}$ which have the identical size for the $Y$ component and two prediction error subimages with an identical size, i.e., $\mathrm{PEM}_{2}^{1}$ and $\mathrm{PEM}_{2}^{2}$ for either the $C_{b}$ or $C_{r}$ components. Each macroblock in the second highest priority detail subimages may contain the picture information for these seven prediction error subimages and their associated motion vectors, disparity vectors, and disparity-compensated prediction errors. Thus, the expression of $\overline{C \text { Slice }}$ for the second highest priority detail subimages is defined as follows:

$$
\begin{aligned}
\overline{C \text { Slice }}= & \overline{C \text { Slice } h}+\overline{C M B h} \times \bar{n}_{M B}^{(2)}+\overline{C M V} \times \bar{n}_{m v}^{(2)} \\
& +\overline{C D I S}{ }^{(2)} \times \bar{n}_{D I S}^{(2)}+\sum_{j=1}^{3} \overline{P E M_{4}^{j}(Y)} \\
& +\sum_{j=1}^{2} \overline{P E M_{2}^{j}}\left(C_{b}\right)+\sum_{j=1}^{2} \overline{P E M_{2}^{j}}\left(C_{r}\right) \\
& +\sum_{j=1}^{3} \overline{P E D_{4}^{j}}(Y)+\sum_{j=1}^{2} \overline{P E D_{2}^{j}}\left(C_{b}\right) \\
& +\sum_{j=1}^{2} \overline{P E D}_{2}^{j}\left(C_{r}\right)
\end{aligned}
$$

where $\overline{C M V}^{(2)}$ and $\overline{C D I S}(2)$ are, respectively, the average numbers of bits in a coded motion vector and a coded disparity vector for the second highest priority subimages. $\bar{n}_{M B}^{(2)}, \bar{n}_{m v}^{(2)}$, and $\bar{n}_{D I S}^{(2)}$ are the average numbers of macroblocks, motion vectors, and disparity vectors in a slice, respectively. $\overline{P E M}_{m}^{j}$ and $\overline{P E D}_{m}^{j}$ denote, respectively, the average numbers of bits in the motion-compensated and disparity-compensated prediction error subimages belonging to a slice. Similarly, the expression of $\overline{C \text { Slice }}$ for the third highest priority detail subimages with disparity information is given by

$$
\begin{aligned}
\overline{\text { CSlice }}= & \overline{\text { CSliceh }}+\overline{C M B h} \times \bar{n}_{M B}^{(3)}+\overline{C M V} \\
& \times \bar{n}_{m v}^{(3)}+\overline{C D I S}(3) \times \bar{n}_{D I S}^{(3)} \\
& +\sum_{j=1}^{2} \overline{P E M_{2}^{j}}(Y)+\sum_{j=1}^{2} \overline{P E D}_{2}^{j}(Y) .
\end{aligned}
$$



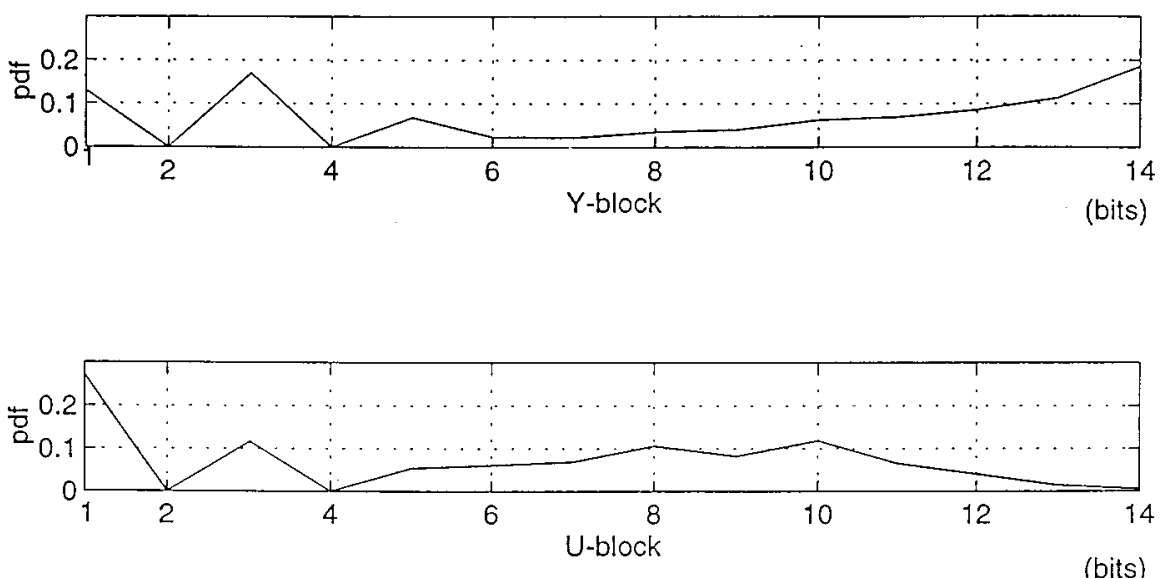

(bits)

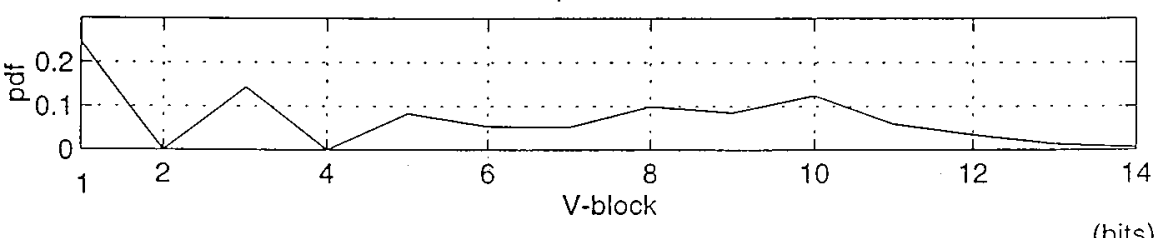

(bits)

Fig. 8. Data distributions of dc coefficients in $\mathrm{Y}, \mathrm{U}$, and $\mathrm{V}$ blocks in the I frame generated from the low-resolution subimage in the left image sequence.

Note that the lowest priority subimages are not considered in the calculation of both the $\overline{\text { Slice }}$ and $\overline{\text { CSlice }}$ because they are dropped prior to the transmission.

\section{B. Adaptive FEC Code Combining Strategy for Outer Code Combining}

This section presents an adaptive FEC code combining scheme which is able to achieve the optimal outer code selection according to the statistical data distribution of the multiresolution 3-D stereo video source coding. This combining strategy is similar to the concept of Huffman source coding. A typical color 3-D image sequence "Spiral Ball" (size 640 $\times 480$ ), in the 3-D library of the 3-D museum constructed by Multimedia Creators Network of Pioneer Electric Corporation, is considered in the design of outer code selection. Two basic shortened $\mathrm{BCH}$ codes of $(7,4)$ and $(15,7)$ are chosen as the basic core codes in the outer code combining to minimize the code overhead and also to provide good protection with reasonable delay. A number of low-resolution enhanced I, P, and B frames with disparity information generated from both the left and right image sequences in Spiral Ball using the MPEG-based joint motion/disparity-compensated wavelet multiresolution coding scheme are treated as a typical test video pattern to evaluate the performance of outer code combining. For the sake of describing the characteristics of the low-resolution enhanced frame clearly, we investigate its two essential components, i.e., a pure low-resolution frame generated from the low-resolution subimage in only the left image sequence and disparity information, separately. Fig. 8 shows the data distributions of dc coefficients in $\mathrm{Y}, \mathrm{U}$, and $\mathrm{V}$ blocks of the pure low-resolution I frames (without disparity information), respectively. On the other hand, Fig. 9 illustrates the data distribution for the disparity vectors that are obtained from the low-resolution subimages in both the left and right image sequences when the I frame is generated from the left image sequence at a specfic time instant. It should be noted that the above data distributions are evaluated over all the left and right image frames in the Spiral Ball. Similarly, the data distributions of high-priority data (MB header and motion vectors) in the pure low-resolution $\mathrm{P}$ and $\mathrm{B}$ frames generated from the left image sequence and their associated disparity vectors are illustrated in Figs. 10 and 11, respectively. The following procedure for encoding $\mathrm{dc}_{Y}$ in the pure low-resolution I frame is treated as a typical example for outer code combining: 1) if $1 \leq \mathrm{dc}_{Y} \leq 4$ bits, use $\mathrm{BCH}(7,4)$ to encode $\left.\mathrm{dc}_{Y} ; 2\right)$ if $5 \leq \mathrm{dc}_{Y} \leq 7$ bits, use $\mathrm{BCH}(15,7)$ to encode it; 3$)$ if $8 \leq \mathrm{dc}_{Y} \leq 11$ bits, use $\operatorname{BCH}(7,4)+\mathrm{BCH}(15,7)$ to encode it; and 4) if $12 \leq \mathrm{dc}_{Y} \leq 14$ bits, use $2 \times \mathrm{BCH}(15,7)$ to encode it. Note that the maximum number of bits in $\mathrm{dc}_{Y}$ is 14 . The probability for each data range can be found as: 1) $\operatorname{Pr}\left\{1 \leq \mathrm{dc}_{Y} \leq 4\right\}=0.3$;2) $\operatorname{Pr}\left\{5 \leq \mathrm{dc}_{Y} \leq 7\right\}=0.1125$; 3) $\operatorname{Pr}\left\{8 \leq \mathrm{dc}_{Y} \leq 11\right\}=0.205$; and 4) $\operatorname{Pr}\left\{12 \leq \mathrm{dc}_{Y} \leq 14\right\}=0.3825$. Therefore, the average number of bits in the $\mathrm{BCH}$ outer coded $\mathrm{dc}_{Y}$ belonging to the I frame becomes 19.7725 .

Similarly, the same $\mathrm{BCH}$ outer code combining can be applied to dc values in both the $\mathrm{U}\left(C_{b}\right)$ and $\mathrm{V}\left(C_{r}\right)$ blocks belonging to a pure I frame generated from the left low-resolution subimage. Hence, $\overline{C D C}_{U}=15.3707$ and $\overline{C D C}_{V}=15.2443$. $\bar{n}_{Y}, \bar{n}_{U}, \bar{n}_{V}$ are found to be 40,10 , and 10 , respectively. A combining of $6 \times \mathrm{BCH}(15,7)$ is used to encode a fixed 38-bit slice header with high FEC protection for the I frame. Similarly, a fixed 2-bit $\mathrm{MB}$ header is protected by $\mathrm{BCH}(7,4)$. As a result, $\overline{C \text { Slice } h}=90$ and $\overline{C M B h}=7$. 


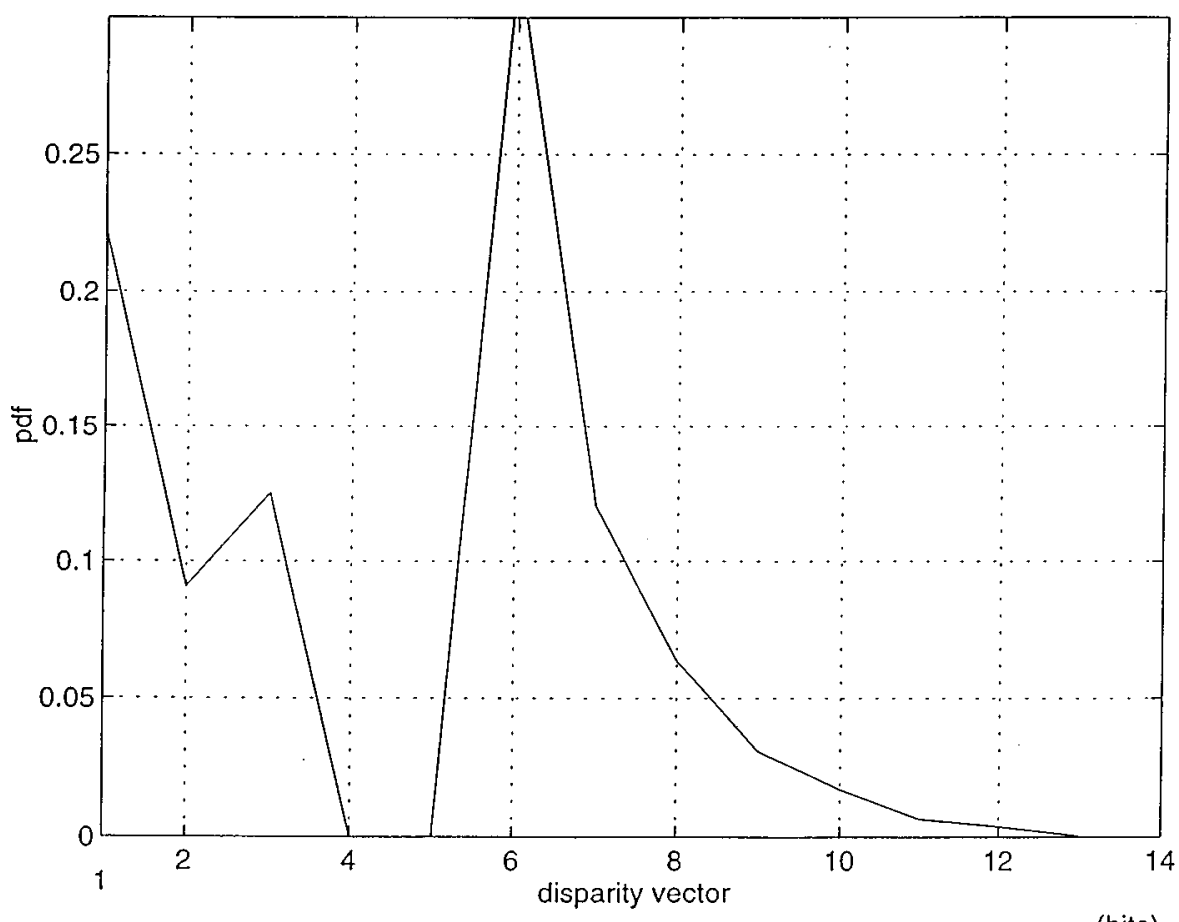

Fig. 9. Data distribution of disparity vector obtained from the low-resolution subimages in both the left and right image sequences when I frame appears in the left image sequence.
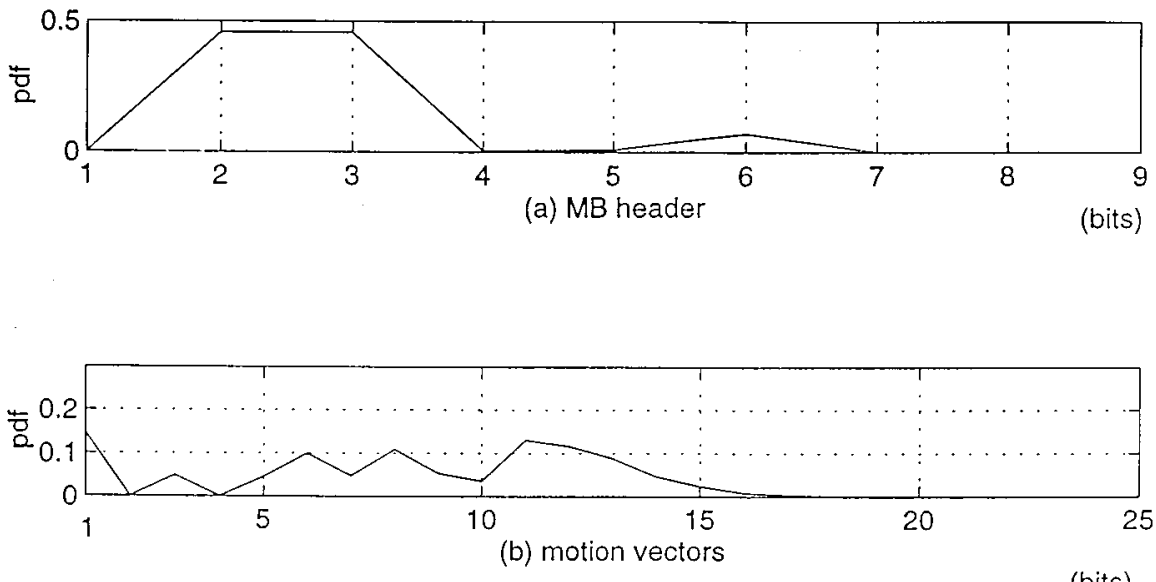

(bits)

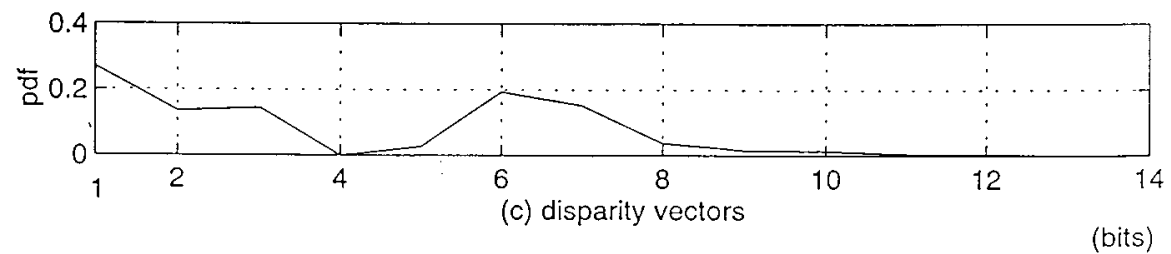

Fig. 10. Data distributions of MB header, motion vectors, and disparity vectors in the low-resolution enhanced $\mathrm{P}$ frame.

In addition, $\bar{n}_{M B}=10$ and $\overline{A C}=6968.57$. For the disparity information, $\overline{C D I S}=12.386, \bar{n}_{D I S}=160$, and $\overline{P E D}_{4}(Y)+\overline{P E D}_{2}\left(C_{b}\right)+\overline{P E D}_{2}\left(C_{r}\right)=5591.733$. From (13), $\overline{C \text { Slice }}=15800.17$. Moreover, the average code rate for the ATM cell containing the low-resolution enhanced I frame with disparity information is computed by (12). It yields
$\bar{R}_{c, I}=0.62$. For the low-resolution enhanced $\mathrm{P}$ and B frames, the same $\mathrm{BCH}$ outer code combining scheme is applied to their high-priority data according to their data distribution. However, a combining of $\mathrm{BCH}(15,7)+2 \times \operatorname{BCH}(31,16)$ is applied to the 38-bit slice header of either $\mathrm{P}$ or $\mathrm{B}$ frames with a medium FEC protection. This leads to $\overline{C \text { Slice } h}=77$. The average code rates 


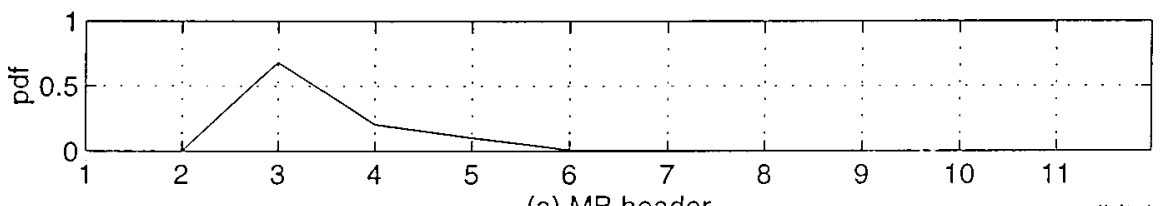

(a) MB header

(bits)

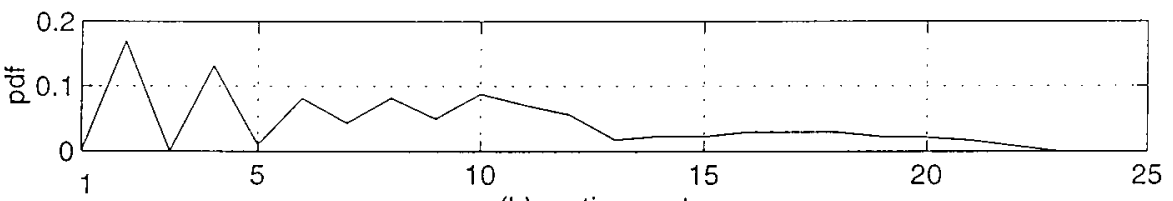

(b) motion vectors

(bits)

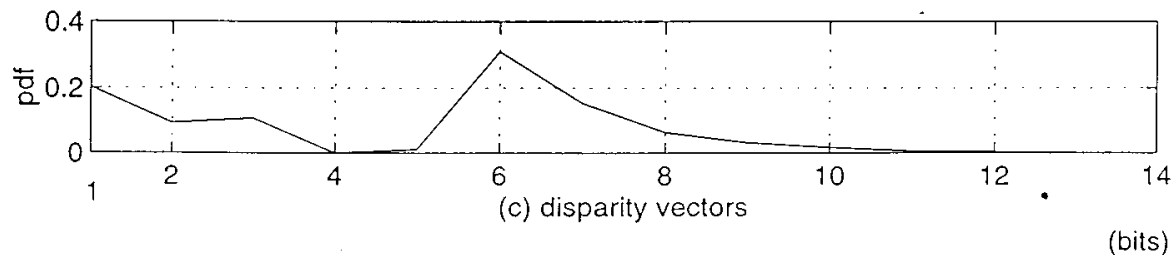

Fig. 11. Data distributions of $\mathrm{MB}$ header, motion vectors, and disparity vectors in the low-resolution enhanced $\mathrm{B}$ frame.

for the ATM cells containing the low-resolution enhanced $\mathrm{P}$ and $\mathrm{B}$ frames are found to be $\bar{R}_{c, P}=0.69$ and $\bar{R}_{c, B}=0.65$, respectively. The outer FEC code combining based on these two basic $\mathrm{BCH}$ codes of $(7,4)$ and $(15,7)$ can be also applied for the error protection of either the second or third highest priority detail subimages. This would yield $\bar{R}_{C}^{(2)}=0.767$ for the second highest priority detail subimages and $\bar{R}_{C}^{(3)}=0.8$ for the third highest priority one.

\section{TRANSMission OF MUltiPle VideOS OVER ATM-BASED Multicode CDMA MobILE COMMUNiCATION NETWORKS}

In the near future, the third-generation mobile communication will allow high-mobility users to access video information stored at various network sites. The multicode CDMA seems one of the major technologies [14], [15] for supporting video services in the third-generation system. In this paper, we are trying to propose a generic multicode CDMA system for providing the transmission of multiple videos via wireless ATM network. For the applications of 3-D stereo videos, Fig. 12 illustrates the wireless ATM-based multicode CDMA architecture for the transmission of MPEG-based multiresolution 3-D video. Four FEC channel coding schemes are considered in the ATM cell packetizer for the MPEG-based wavelet multiresolution 3-D video coding. The FEC code with high-error protection is applied to ATM headers. The adaptive outer/inner FEC code combining scheme with variable error protection is used to protect the low-resolution subimages, motion vectors, and disparity vectors according the priority analysis. The same code combining mechanism is also applied to the detail subimages. For example, two outer FEC codes with medium- and low-error protection in the code combining scheme are used to protect the medium- and low-priority detail subimages, respectively. The architecture illustrated in Fig. 12 with a little modification is also vaild for different types of video coding schemes, i.e., MPEG-4, H.263, ‥ etc. However, the traditional CDMA system may not be sufficient to accommodate the transmission of the high-bit-rate videos via a narrow-band mobile channel. In the multicode CDMA system, when the $k$ th video needs $N_{k}$ times the basic transmission rate, it converts the original high-bit-rate bit stream using a multiplexer or serial-to-parallel converter into $N_{k}$ basic rate streams, encodes each with a different spreading code, modulates them with a different Walsh modulator, and superimpose them before upconverting for mobile transmission. In other words, each spreading code in the multicode CDMA carries a data stream with a basic rate $r_{B} \cdot N_{k}$ spreading codes in parallel will provide a single user with $N_{k}$ times the basic rate capability. Note that each video admitted into the system has a primary PN code assigned to it. These PN codes are not orthogonal between different video users. To avoid the self-interference that a video user employing multiple codes may incur, the multiple codes to/from one user should be made orthogonal. If $c_{k}$ is the primary PN code of video $k$ with a basic transmission bit rate $r_{B}, N_{k}$ new concatenated spreading codes, $a_{k m}$ can be derived by concatenating $c_{k}$ with $d_{m}, 1 \leq m \leq N_{k}$, where $d_{m}$ is the $m$ th orthogonal code and $d_{l} \perp d_{m}$ if $\bar{l} \neq m$. It should be mentioned that the actual value of $N_{k}$ depends on the frame type, frame period, basic transmission rate, desired cell loss, and total number of bits in all the coded ATM cells. A spreading code assignment mechanism is proposed to determine an appropriate value of $N_{k}$ which is able to achieve both the higher transmission capability and better received picture quality. Due to the orthogonality requirement, the maximum number of orthogonal codes per user is the 

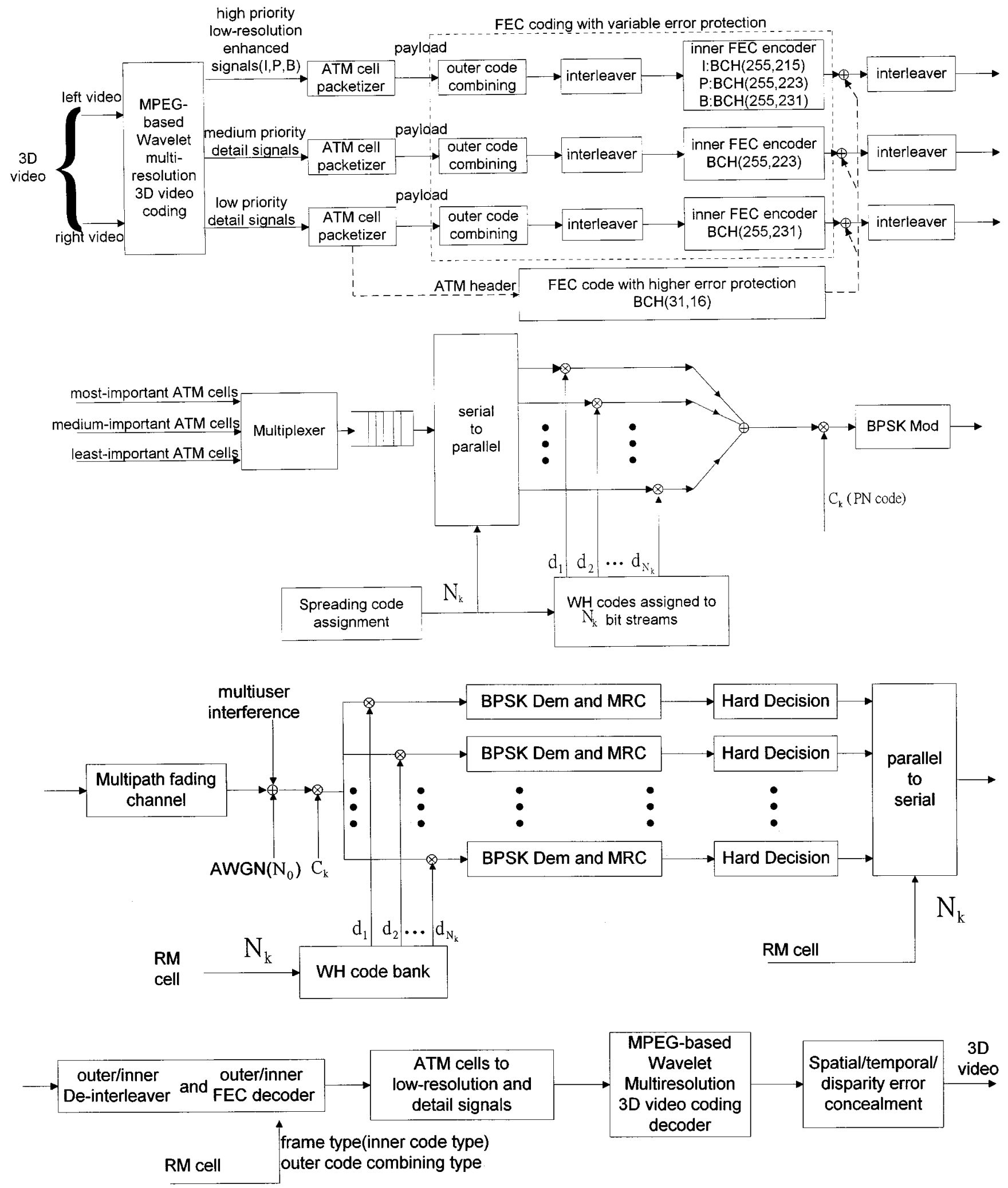

Fig. 12. Overall schematics of MPEG-based 3-D video transmission via ATM-based multicode CDMA system resource management (RM) cell contains Nk, frame type, and outer code combining type. Note that the inner BCH codes may be replaced by either turbo or RCPC codes.

ratio $N_{c}$ between the channel chip rate and the Walsh modulator output rate. $N_{c}$ is termed as the spreading sequence length. Hence, $N_{k} \leq N_{c}$. The above coding strategy is called the subcode concatenated scheme that orthogonal sequences are con- catenated with a PN sequence to increase the randomness of the orthogonal sequence. The binary orthogonal sequences used in this paper were the well-known Walsh-Hadamard (WH) codes which have zero cross correlation at zero time delay. They are 
used when synchronization of transmission can be maintained. Unfortunately, the multipath fading in a cellular radio environment introduces nonzero time delays that destroy the orthogonality between WH codes. Fong et al. [15] showed that a sufficiently long PN sequence is concatenated with WH codes to randomize and eliminate their unsatisfactory and inhomogenous behavior at nonzero time delays. The long PN sequence may be chosen as either an $m$ sequence or Gold sequence.

\section{A. Multicode CDMA Transmitter Model with Concatenated Orthogonal/PN Spreading Code Scheme}

For $K$ videos transmitted over a mobile channel, each of them is divided into $N_{k}(t)$ parallel data streams (virtual channels) for user $k$ at time $t$, where a specific WH code is assigned to each virtual channel. The value of $N_{k}(t)$ is dynamically determined by a code assignment mechanism discussed in the next section. For simplicity, $N_{k}(t)$ is assumed to be a constant $N_{k j_{k_{k}}}$ in the $j_{k}$ th time interval $I_{k j_{k}}=\left[T_{k j_{k}}, T_{k, j_{k}+1}\right)$, i.e., $N_{k}(t)=N_{k j_{k}}$ for $t \in I_{k j_{k}}$. Hence, the total number of $W H$ codes assigned to all the $K$ users in the new time interval $I_{j}$ is $M=\sum_{k=1}^{K} N_{k j_{k}}$ where $I_{j}$ denotes the $j$ th intersection interval of the time intervals for all the $K$ videos, i.e., $I_{j}=\cap_{k=1}^{K} I_{k j_{k}}$. Usually, $T_{f}=T_{k, j_{k}+1}-T_{k, j_{k}}$ is a fixed value called the frame period. Note that WH codes assigned to different users may be identical. It should be mentioned that the above arguments are valid when each user has a continuous video presentation. However, this assumption may not be true since some users may not have videos during a specific time interval, i.e., $I_{k j_{k}}$. To tackle this difficulty, the value of $N_{k j_{k}}$ for this particular interval is set to zero. Therefore, the above argument become valid again. For simplicity, we assume that each of the $K$ videos has its corresponding picture frames during $I_{j}$. The transmitting BPSK signal $s_{k m}(t)$ of the $m$ th data stream (virtual channel) belonging to the $k$ th video during the $j$ th time interval $I_{j}$ is expressed as

$$
\begin{aligned}
& s_{k m}(t)=\sqrt{2 P} a_{k m}(t) b_{k m}(t) \cos \left(\omega_{c} t+\theta_{k m}\right), \\
& t \in I_{j}, 1 \leq m \leq N_{k j} \leq N_{c}, 1 \leq k \leq K
\end{aligned}
$$

where $P$ is the transmission power of the base station, $\theta_{k m}$ is the random phase angle, uniformly distributed between 0 and $2 \pi$, introduced by the modulator, and $b_{k m}(t)$ is the data signal which consists of a sequence of rectangular pulses of duration $T$, i.e.,

$$
b_{k m}(t)=\sum_{i=-\infty}^{\infty} b_{k m}^{(i)} \Pi_{T}(t-i T)
$$

where $b_{k m}^{(i)} \in\{1,-1\}$ and $\Pi_{T}(t)$ is a unit pulse function of duration $T$. The concatenated spreading code $a_{k \cdot m}(t)$ can be expressed as

$$
a_{k m}(t)=\sum_{i=-\infty}^{\infty} a_{k m}^{(i)} \Pi_{T_{c}}\left(t-i T_{c}\right)
$$

where $a_{k m}^{(i)} \in\{-1,1\}$ is the concatenated spreading sequence which is equal to the product of a PN sequence $c_{k}^{(i)} \in\{-1,1\}$ used by the $k$ th user and a $W H$ code sequence $d_{m}^{(i)} \in\{-1,1\}$ assigned to its $m$ th virtual channel. The duration of each data bit is $T$, while the duration of each chip in the spreading code is $T_{c}$. The number of chips per bit is $N_{c}=T / T_{c}$, where $N_{c}$ is an integer. The period of the WH code sequence $d_{m}^{(i)}$ is equal to the processing gain $N_{c}$. The long PN sequence $c_{k}^{(i)}$ has a period, $N_{P N}$, that is much greater than $N_{c}$. Moreover, $N_{P N}$ and $N_{c}$ are chosen to be relatively prime so that every possible chip of the PN sequence can occur at the beginning of some data bit. As a result, the total signal transmitted to $K$ users is

$$
s(t)=\sum_{k=1}^{K} \sum_{m=1}^{N_{k j}} s_{k \cdot m}(t), \quad t \in I_{j}=\bigcap_{k=1}^{K} I_{k j} .
$$

\section{B. Mobile Radio Channel Model}

The Rayleigh multipath fading model is the general accepted channel model for mobile communications [27]. In this paper, we adopted the Rayleigh fading model for performance analysis in our multicode CDMA system. The channel impulse response for the $m$ th virtual channel of the $k$ th user is given by

$$
\begin{aligned}
h_{k m}(t)=\sum_{l=1}^{L_{k m}} \beta_{l k m} \delta\left(t-\tau_{l k m}\right) e^{j \phi_{l k m}}, & \\
& t \in I_{j}, 1 \leq m \leq N_{k j}, 1 \leq k \leq K
\end{aligned}
$$

where $\beta_{l k m}$ is the $l$ th Rayleigh distributed random path gain, $\phi_{l k m}$ is the $l$ th random path phase, uniformly distributed between zero and $2 \pi$, and $\tau_{l k m}$ is the $l$ th uniformly distributed random delay ranging from zero to one data bit period $T . \delta(t)$ represents the unit impulse function. $L_{k m}$ denotes the number of resolvable multipaths for the $m$ th virtual channel of user $k$. In addition, it should be mentioned that these channel parameters vary with the transmitter-receiver distance. It may be shown that $\bar{\beta}_{l k m}^{2}=\bar{\beta}_{l k 11}^{2}=\bar{\beta}_{l k}^{2}$ and $L_{k m}=L_{k 1}=L_{k}$ for $1 \leq m \leq N_{k j}$ since all the parallel virtual channels introduced by the same user are transmitted over the same propagation environment between the transmitter and receiver and then would have identical channel characteristics, where $\bar{x}^{2}$ denotes the variance of a random variable $x$.

\section{Receiver Model}

The received signal at the input to the matched filter in the mobile receiver is given by

$$
\begin{aligned}
r(t)= & \operatorname{Re}\left\{\int_{-\infty}^{\infty} h_{k m}(\tau) \tilde{s}_{k m}(t-\tau) \exp \left(j \omega_{c} t\right) d \tau\right\} \\
& +n(t) \\
= & \sqrt{2 P} \sum_{k=1}^{K} \sum_{m=1}^{N_{k j}} \sum_{l=1}^{L_{k m}} \beta_{l k m} a_{k \cdot m}\left(t-\tau_{l k m}\right) \\
& \cdot b_{k m}\left(t-\tau_{l k m}\right) \cos \left(\omega_{c} t+\varphi_{l k m}\right)+n(t) \\
\varphi_{l k m}= & -\omega_{c} \tau_{l k m}+\phi_{l k m}+\theta_{l k m}
\end{aligned}
$$

where $\tilde{s}(t)$ is the complex envelope of $s(t), \operatorname{Re}\{\cdot\}$ denotes the real part of complex number, and $n(t)$ is a white Gaussian noise with two-sided power spectral density $N_{0} / 2$.

For simplified analysis, the first virtual channel of the first user is chosen as the reference for calculating the probability of error of its data symbol $b_{11}^{(j)}$ in the $j$ th sampling time interval $I_{s}^{(j)}=[(j-1) T, j T]$. The receiver is able to coherently recover the carrier phase $\varphi_{l k m}$ and $\tau_{k l m}$ locking to the $l$ th path as 
a reference path between the transmitter and its corresponding receiver. All other paths constitute interference. That is, we assume without loss of generality that $\varphi_{l 11}=0$ and $\tau_{l 11}=0$. The envelope of the matched-filter output at the $j$ th sampling time instant $(t=j T)$ is denoted by $Y_{11}^{(j)}$ and can be expressed as

$$
\begin{aligned}
Y_{11}^{(j)} & =\int_{(j-1) T}^{j T} r(t) a_{11}(t) \cos \left(\omega_{c} t\right) d t+\nu \\
& =\beta_{l 11} \sqrt{\frac{P T^{2}}{2}} b_{11}^{(j)}+\text { Int }_{1}+\text { Int }_{2}+\text { Int }_{3}+\nu \\
I_{s}^{(j)} & \subseteq I_{j}=\bigcap_{k=1}^{K} I_{k j}
\end{aligned}
$$

where

$$
\nu=\int_{(j-1) T}^{j T} n(t) a_{11}(t) \cos \left(\omega_{c} t\right) d t
$$

$\mathrm{Int}_{1}$ is the intramultiuser interference (self-interference) indicating the interference introduced by the other virtual channels of reference user, $\mathrm{Int}_{2}$ is the intramultipath interference, and $\mathrm{Int}_{3}$ denotes the inter multiuser interference. Note that the orthognality of the concatenated spreading codes eliminates the intramultiuser interference (self-interference) [15]. This leads to $\mathrm{Int}_{1}=0$.

\section{Calculation of Average Cell Loss Rate}

This wireless ATM cell is transmitted over the wireless CDMA link. Assuming that bit synchronization and cell synchronization have been achieved, the mobile receiver first decodes the ATM header. If the cell is successful, the receiver then decodes the payload. There are two major factors that cause wireless networks to discard ATM cells. One is bit errors in the header due to the multipath fading, interference, and channel noise. The other is buffer overflow in multiplexing or cross-connecting equipment. The cell loss process due to buffer overflow can be approximated by a two-state Markov chain called the Gilbert model [28]. Here, for simplicity, we only consider the cell loss due to the bit errors on the header. Usually, a single cell loss may cause a loss of an amount of image data and results in a high degradation of picture quality. Moreover, the picture is still corrupted by the multipath fading, interference, and noise even if the ATM cell is successful. Fortunately, this picture degradation can be eliminated by using the proposed FEC code combining strategy, spatiotemporal filtering [31], and spatial/temporal error concealment techniques. Therefore, the cell loss rate (CLR) is regarded as the major performance index used to evaluate the quality of received picture. For the simplified derivation of the CLR, the Gaussian assumption is to take all the self-interference, intramultipath interference, and intermultiuser interference terms as Gaussian noise. In addition, since all the signals including the desired signal and the interfering signals caused by the other $(K-1)$ users relative to reference user (user 1) are transmitted to the first mobile receiver from the same base station (downlink) and have the identical propagation environment between the base station and the receiver for user 1 , it can be shown that $\bar{\beta}_{q k}^{2}=\bar{\beta}_{q 1}^{2}$ and $L_{k}=L_{1}=L$. Let $\bar{E}_{b}\left(=\bar{\beta}_{l}^{2} P T\right)$ represent the receivied signal energy per bit via the $l$ th path (reference path).
Thus, the received signal is found to be $T \bar{E}_{b} / 2$. Moreover, according to the orthogonality of the concatenated spreading codes, it can be found that the variance of the total interfering signals is $\left(T \bar{E}_{b} \kappa^{2} M / 4\right) \times\left[\eta-\left(N_{1 j} / M\right)\right]+\left(N_{0} T / 4\right)$, where

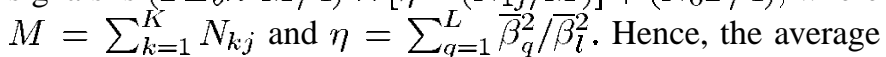
value of half the signal-to-noise plus interference power ratio becomes

$$
\begin{aligned}
\bar{\gamma}_{b} & =\frac{1}{2} \frac{\frac{T \bar{E}_{b}}{2}}{\frac{T \bar{E}_{b} \kappa^{2} M}{4} \times\left[\eta-\frac{N_{1 j}}{M}\right]+\frac{N_{0} T}{4}} \\
& =\left[\frac{2 M}{3 N_{c}}\left(\eta-\frac{N_{1 j}}{M}\right)+\frac{N_{0}}{\bar{E}_{b}}\right]^{-1} .
\end{aligned}
$$

Note that $\eta$ is always greater than one. For simplicity, assume that the path gains for all the virtual channels in the same user are independent identically distributed (i.i.d.), i.e., $\bar{\beta}_{q}^{2}=\bar{\beta}^{2}$, $1 \leq q \leq L$. Thus, the value of $\eta$ is identical to $L$.

Proakis [27] showed that the BER of a receiver with maximal ratio combining (MRC) of order $L_{d}$ can be expressed as a form in terms of $\bar{\gamma}_{b}$

$$
\begin{aligned}
p_{e}= & \left(\frac{1-\mu}{2}\right)^{L_{d}} \times \sum_{s=0}^{L d-1}\left(\begin{array}{c}
L_{d}-1+s \\
s
\end{array}\right)\left(\frac{1+\mu}{2}\right)^{s} \\
& \text { for MRC of order } L_{d} .
\end{aligned}
$$

In (26), for maximal ratio combining, the quantity $\bar{\gamma}_{b}$ represents the average signal-to-noise ratio per combined path, and $\mu=\sqrt{\bar{\gamma}_{b} / 1+\bar{\gamma}_{b}}$.

Since a BCH code of $(31,16,3)$ is proposed to protect the 16-bit compressed ATM header, the CLR is computed by

$$
\mathrm{CLR}=\sum_{i=4}^{31}\left(\begin{array}{c}
31 \\
i
\end{array}\right)\left(1-p_{e}\right)^{31-i} p_{e}^{i} .
$$

\section{E. Determination of Average WH Code Number for 3-D Stereo Video}

The average number of WH codes assigned to a specific picture frame in conventional 3-D stereo video is dependent on the average number of bits in a picture frame $\bar{Q}_{f}$, the transmission bit rate for a virtual channel (WH code) $r_{B}$, and the frame period $T_{f}$. Thus, the average $\mathrm{WH}$ code number assigned to the specific frame is estimated by

$$
\bar{N}=\left\lceil\frac{\bar{Q}_{f}}{r_{B} \bar{R}_{c} T_{f}}\right\rceil .
$$

However, the expression of (28) can be extended to the MPEG-based multiresolution 3-D video coding. From Section IV, it is known that there are three different ATM code rates, i.e., $\bar{R}_{C, I}, \bar{R}_{C, P}$, and $\bar{R}_{C, B}$ for the low-resolution enhanced I, $\mathrm{P}$, and $\mathrm{B}$ frames with disparity information generated from the low-resolution subimages in 3-D video at three different time points. $\bar{Q}_{I}, \bar{Q}_{P}$, and $\bar{Q}_{B}$ correspond to the average numbers of bits in the low-resolution enhanced I, P, and B frames including both the disparity vectors and disparity-compensated prediction errors, respectively. On the other hand, $\bar{R}_{C}^{(2)}$ and $\bar{R}_{C}^{(3)}$ are, respectively, the ATM code rates for the second and third highest priority detail subimages. The average total number of bits in either the second or third priority detail subimages containing 
the disparity information is $\bar{Q}^{(2)}$ or $\bar{Q}^{(3)}$. As a result, the average $\mathrm{WH}$ code number assigned to a $3-\mathrm{D}$ enhanced picture frame at a specific time instant is calculated by

$$
\bar{N}_{x}=\left[\frac{\frac{\bar{Q}_{X}}{\bar{R}_{C, X}}+\frac{\bar{Q}^{(2)}}{\bar{R}_{C}^{(2)}}+\frac{\bar{Q}^{(3)}}{\bar{R}_{C}^{(3)}}}{r_{B} T_{f}}\right]
$$

where $X=I, P$, or $B$.

More details of the spreading code assignment mechanism can be found in (38).

\section{SPATIAL, TEMPORAL, AND DiSPARITY ERROR CONCEALMENT TECHNIQUES FOR 3-D STEREO VIDEO WITH INFORMATION LOSS}

In this section, we propose the cell loss resilience techniques to conceal the information loss in 3-D stereo video due to multipath fading and interference. For the multiresolution 3 -D video coding, the low-resolution subimage in the left image sequence plays an essential role in the picture quality of received 3-D video since the high-frequency detail subimages are visually less important than the low-resolution subimage and have very little impact on video quality. The low-resolution subimage in the right image subimage may be reconstructed by the disparity-compensated technique. In this paper, the low-resolution subimage in the left image sequence is encoded by using MPEG-2 coding technique. However, Zhang and Lee [17] have shown that the MPEG-2 source coding algorithm is very sensitive to multipath fading channel and interference. For example, a single bit error in the ATM header (cell loss) will often result in loss of a whole image block, which could further cause consecutive block losses. Sometimes, a number of consecutive blocks may be destroyed when a single bit error occurs in either the slice header or macroblock header or motion vector, even though the ATM cell is successful. On the other hand, the ATM cell loss may result in loss of a whole image block in the right image when the cell contains the disparity vector or the control headers related to the disparity.

The cell discarding may also be caused by network congestion and buffer overflow [28]. Several methods have been proposed to minimize the effect of cell loss on the received image quality. These schemes include FEC coding with interleaving and error concealment using postprocessing methods [18]-[22]. In this paper, we consider the combination of these strategies. The two main cell loss resilience techniques described here are the spatial and temporal error concealment techniques. The spatial error concealment exploits the spatial redundancy in one picture. This technique is proposed for intracoded pictures, i.e., I frame, where no motion information exists. Temporal error concealment makes use of the temporal redundancy in one sequence. It is devoted to intercoded pictures, i.e., $\mathrm{P}$ and $\mathrm{B}$ frames, because there exists some motion information. The temporal error concealment is also suitable for the recovery of the damaged right image with respect to the left image when the temporal information is replaced by the disparity information. It should be mentioned that the spatial/temporal/disparity error concealment techniques are also applied to the recovery of information loss, even though the ATM cells are successful. For instance, a single bit error in the DCT coefficients may damage one block when the DCT coefficients of each block are coded using run-length and variable-length coding. Moreover, a number of subsequent blocks may be destroyed if a bit error occurs in the macroblock header. Note that the information loss in the detail subimages caused by ATM cell loss did not result in the severe image quality degradation. It only leads to the blurred image quality.

\section{A. Multiresolution Multidirectional Interpolation for Spatial Error Concealment}

Since lost blocks are often correlated with neighboring spatial blocks due to the correlation property of nature images, interpolation and surface fitting can be used to partially recover the lost information [18], [19]. However, such techniques are low-pass filtering in nature and tend to ignore high-frequency information such as edges and contours. Lee et al. [21] have proposed a fuzzy logic approach to recover the lost blocks with high frequency and yield the desired reconstructed picture quality. Meanwhile, this fuzzy loss recovery technique is too complicated for implementation. Therefore, a cost-effective multidirectional interpolation [20] is employed to conceal the lost block with high frequency, where the damaged block is surrounded by eight neighboring blocks of correctly received pixels. A voting classification mechanism operates on the eight neighboring blocks and determines which directions characterize the three strongest edges. A spatial interpolation is performed to create the recovered block for each direction separately. Those interpolation results are then mixed by averaging to obtain the final recovered block containing multiple interpolated edges. The determination of the directions for the strongest edges depends on extending edges present in the neighboring blocks so that they pass through the damaged block. The direction classification for edges can be performed by a Sobel gradient operator which is able to give accurate angle estimates.

The multidirectional interpolation works well when the angular direction of edge is near to one of eight directional categories. However, this method becomes invalid when the edge direction is not close to any directional category. To tackle this difficulty, a multiresolution direction classification is proposed to achieve the more accurate direction estimation. This classification is a two-level windowing process. The first-level process is the original voting mechanism which provides a coarse estimation for the edge direction and then determines the selected nested region between two adjacent direction categories. The second-level process is a fine estimation for the edge direction based on four finer directional categories belonging to the chosen nested region. The difference between any two adjacent finer directional categories is $5.625^{\circ}$. In simulation results, we will compare our multiresolution approach with the original one and show the improvement of our method.

Sometimes, a cell loss may cause a sequence of consecutive horizontal block losses when it contains a slice header, a macroblock header, or a sequence of block data. A sliding block iteration method is proposed to conceal the continguous block loss by using the multiresolution multidirectional interpolation block by block. First, the damaged left-most block 0 is recovered 
based on its seven neighboring correct blocks. Next, the recovered block is treated as the correct neighboring block adjacent to the second left-most damaged block and can be applied for multidirection interpolation. In the same time, the similar recovery process is performed from the right-most damaged block to the left one. The sliding block iteration is terminated when all the damaged blocks have been recovered. The method is also valid for color MPEG videos by applying the iteration to each of the Y, $\mathrm{U}$, and $\mathrm{V}$ blocks independently. Another method to conceal consecutive block losses is to apply the block-interleaving scheme to isolate the block loss. However, it may cause encoding and reconstruction delay [22], [29]. Sometimes, it will become invalid when the consecutive block losses are caused by the bit errors in either the slice header or macroblock header. Finally, it should be mentioned that this spatial interpolation is applied for not only the lowest resolution subimage, but also the wavelet reconstruction from the lowest resolution subimage and all the the detail subimages.

\section{B. Iterative Pattern Matching Algorithm for Temporal Error Concealment}

The general approach for temporal error concealment is the temporal replacement [19], [22], [23]. In temporal replacement, damaged blocks in the current frame are replaced by the spatially corresponding ones in the previously decoded reference frame with motion compensation if motion information is available. Narula and Lim [23] have shown that the estimate of motion vector in the lost block (i.e., macroblock of size $16 \times 16$ in MPEG-2) can be obtained by taking the median of all eight adjacent correct motion vectors. Note that the motion vector is defined in a macroblock sense. Therefore, the damaged macroblock can be replaced by a correct or previously concealed macroblock in the reference picture based on the estimate. However, this method does not work well when high-frequency information such as edges, contours, and fine textures appear in the macroblock. To tackle this difficulty, an iterative pattern matching algorithm is proposed to improve the performance of temporal replacement. First, the damaged macroblock is recovered based on the estimated median motion vector. In the spatial domain, the recovered macroblock with $16 \times 16$ pixels is further divided into 16 subblocks with each having $4 \times 4$ pixels. Since the corner subblocks tend to be more correlated with their three immediate neighboring correct subblocks, each corner subblock can be combined with its three neighboring subblocks to construct an estimated larger block of size $8 \times 8$. This estimated block is further replaced by a block in the reference picture which has the minimum mean square error deviation to match the estimated block in the current frame. One can find such a block by using a block matching algorithm [19]. The corner subblocks belonging to the best-matching blocks are then treated as the correct subblocks and can be applied for the next pattern matching. More details of the iterative pattern matching can be found in [35]. The pattern matching process is also valid for the recovery of the macroblocks of B frames. For B frames, two recovered macroblocks may be obtained by using the pattern matching iteration with respect to the previous and the next reference pictures, respectively. Finally, the damaged macroblock of B frame has been concealed by taking the average of these two recovered macroblocks.

The iterative pattern matching can be extended to conceal the consecutive lost macroblocks [35]. The recovery process is performed in the right and left directions simultaneously until all the damaged macroblocks have been recovered.

\section{Iterative Pattern Matching Method for Disparity Error Concealment}

For a 3-D stereo image, the disparity vector is the most important factor in the reconstruction of the right image from the other left image. Thus, a single bit error in the disparity may result in loss of its association image blocks in the right image. The iterative pattern matching method is also valid for the disparity error concealment in the right image when the motion vector is replaced by the disparity vector and the reference picture is the left image. The initially estimated disparity vector is the median of eight correct surrounding disparity vectors. Thus, the damaged image macroblock in the right image may be recovered by a macroblock in the left image shifted by the median disparity vector. In order to improve the recovery performance of the damaged macroblock containing high-frequency information, the initially estimated macroblock should be divided into 16 subblocks. The iterative pattern matching method is used to find the best subblock in the reference left picture to match the initially estimated subblock in the right picture from the corner subblocks to center blocks. Finally, the damaged macroblock can be recovered based on these best matching subblocks.

\section{SimUlation RESULTS}

The channel model described in this paper is identical to Model-I employed in [30]. In this model, the variances of the Rayleigh path gains are the same for all the MPEG users and equal to $-14.2 \mathrm{~dB}$ (0.038). Additionally, the maximum number of resolved multipaths for all the users is four, i.e., $L=4$. The channel noise is set to be $\left(E_{b} / N_{0}\right)=25 \mathrm{~dB}$. The multicode CDMA system has a transmission rate of $r_{B}=64 \mathrm{~kb} / \mathrm{s}$ for each code, a processing gain of $N_{c}=128$, and a bandwidth of $10 \mathrm{MHz}$ [15]. Its corresponding PN and orthogonal spreading sequences are chosen as the Gold sequence with a period of $\left(2^{31}-1\right)$ and the WH code with a period of 128 , respectively. For the CDMA receiver, it has a maximum ratio combiner (MRC) of order with $L_{d}=4$. For the interleaving scheme, the outer-FEC-coded data are interleaved over 215 bits for I frames (223 bits for $\mathrm{P}$ frames or 231 bits for $\mathrm{B}$ frames) belonging to the lowest resolution subimages or 223 bits for the second priority detail subimages ( 231 bits for the lowest priority detail subimages), and the inner-FEC-coded data plus the coded ATM header data are interleaved over 20 cells.

To evaluate the performance of the MPEG-based wavelet multiresolution 3-D video via a multicode CDMA system, a 3-D stereo video, Spiral Ball, and four two-way H.261 videos are considered as a typical example in the simulation. Both the left and right pictures in 3-D video are decomposed to their lowest resolution subimages and detail subimages by using Daubechie's $W_{6}$ wavelet. ITU (CCITT) Recommendation 


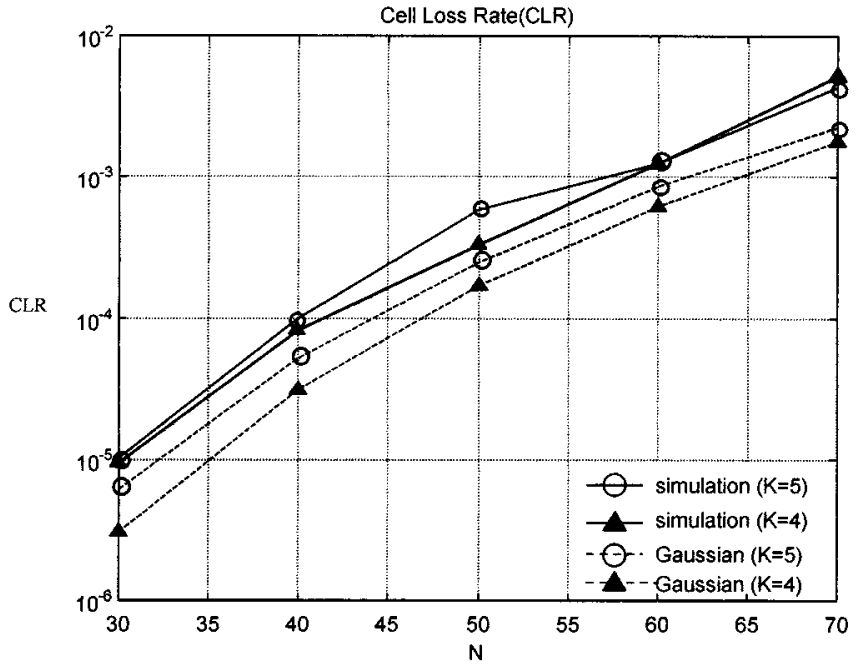

Fig. 13. CLR for 3-D video versus a varying WH code number, $\mathrm{N}$ due to (K-1) interfering two-way H.261 videos, $K=5$ or 4 . Note that solid line with either $\bigcirc$ or $\boldsymbol{\wedge}$ denotes the simulation results whereas dashed line denotes Gaussian approximation.

H.261 is a video compression standard developed to facilitate video conferencing and videophone over the integrated services digital network (ISDN) at $p \times 64 \mathrm{kbps}, p=1,2,3, \cdots$. For example, $64 \mathrm{kbps}(p=1)$ may be appropriate for a one-way videophone service, where the video signal can be transmitted at a rate of $48 \mathrm{kbps}$, and the remaining $16 \mathrm{kbps}$ are used for the audio signal. For a two-way (dual mode) videophone, it is transmitted at a rate of $128 \mathrm{kbps}(p=2)$. Thus, the number of WH codes assigned to a two-way videophone is a equal to two i.e, $N_{H}=2$. From (27), the CLR of a 3-D stereo video due to $(K-1)$ interfering H.261 videos (dual mode) is characterized by the following half the signal-to-noise plus interference power ratio:

$$
\bar{r}_{b}=\left\{\frac{2}{3 N_{c}}\left[3 N+4(K-1) N_{H}+0.0031622\right\}^{-1}\right.
$$

where $N$ is the WH code number assigned to a specific 3-D enhanced picture frame. Note that the definition of 3-D enhanced frame can be found in Section IV. Fig. 13 shows a comparison between the simulation results and Gaussian approximation for the CLR for the 3-D enhanced video picture frame with a varying WH code number, $N$, due to the interfering H.261 videos with either $K=4$ or $K=5$.

The average WH code number assigned to the 3-D enhanced picture frame can be computed by (29) via the evaluation of the average code rate for each low-resolution enhanced frame, the second and third highest priority detailed subimages with disparity information. The average bit counts in the low-resolution enhanced I, P, and B frames belonging to the low-resolution subimages in Spiral Ball (including the disparity and disparity prediction errors) have been found to be $\bar{Q}_{I}=113785.86$ bits per frame, $\bar{Q}_{P}=55109.72$, and $\bar{Q}_{B}=29005.12$, respectively. The average total numbers of bits in the second and third highest priority detail subimages are $\bar{Q}^{(2)}=45887$ and $\bar{Q}^{(3)}=$ 31478 , respectively. From Section IV, it is found that their corresponding ATM code rates are $\bar{R}_{C, I}=0.62, \bar{R}_{C, P}=0.69$, $\bar{R}_{C, B}=0.65, \bar{R}_{C}^{(2)}=0.769$, and $\bar{R}_{C}^{(3)}=0.8$. Thus, by (29), the average WH code number assigned to a 3-D enhanced I frame with disparity information is $\bar{N}_{I}=67$ when the frame period $T_{f}$ is set to $\frac{1}{15} \mathrm{~s}$. Similarly, it may be found that the average WH code numbers assigned to both the 3-D enhanced $\mathrm{P}$ and $\mathrm{B}$ frames are $\bar{N}_{P}=42$ and $\bar{N}_{B}=33$, respectively.

The CLR at a point, $(N, K)=(67,5)$, illustrated in Fig. 13 represents the average CLR with a value of $3.75 \times 10^{-3}$ for the 3-D enhanced I frame due to four interfering H.261 videos. Similarly, the CLR's at $(N, K)=(42,5)$ and $(33,5)$ denote the average CLR's for the 3-D enhanced P and B frames due to four interfering H.261 videos which are equal to $1.87 \times 10^{-4}$ and $2.52 \times 10^{-5}$, respectively. Thus, the average number of ATM cell losses in a 3-D enhanced I frame is equal to 3.101 $\left(=3.75 \times 10^{-3} \times 827\right)$ since the averaged number of ATM cells generated from the 3-D enhanced I frame belonging to Spiral Ball is found to be 827 according to the design methodology of ATM cells proposed in Section IV. The average cell loss numbers in a 3-D enhanced $P$ frame and in a 3-D enhanced B frames are, respectively, found to be 0.072 and $4.88 \times 10^{-3}$ because their corresponding average ATM cell numbers are equal to 383 and 194, respectively. Based on the above analysis, it is seen that the average ATM cell loss number in either 3-D enhanced I, P, or B frames is in (or less than) the order of $\mathrm{O}(1)$.

The second major factor in picture degradation is caused by the transmission error in the payload containing MPEG-based multiresolution 3-D video data, even though its associated ATM cell is successful. The impact of transmission error in MPEG video data generated from the left image sequence seems very significant in the picture quality since both the DCT coefficients and macroblock headers are encoded using run-length coding (VLC). A modification of the original MPEG codec proposed by ISO/IEC is made to localize the error in a picture frame. For instance, a DCT coefficient is lost if a single bit error occurs in that coefficient. A loss of the complete macroblock occurs when a bit error appears in its header. Moreover, a slice of subsequent macroblocks may be destroyed when a bit error occurs in a slice header. For P and B frames generated from the left image sequence, a bit error in a motion vector may result in the corruption of this macroblock and the following predicted macroblocks since motion vectors are differentially encoded for the intercoded macroblocks in the same slice. On the other hand, a number of subsequent macroblocks in the right image may be destroyed when a bit error appears in the disparity vector. The adaptive outer/inner FEC code combining has been proposed to protect the payload in accordance with the importance of data types in MPEG-based multiresolution 3-D videos including either disparity information in the low-resolution subimages or the data belonging to the detail subimages plus motion and disparity-compensated information.

Our system has been evaluated over 200 frames of Spiral Ball. We particularly choose the worst case of an ATM cell loss containing a slice header and a damaged macroblock header in the payload when its associated ATM cell is successful via the transmission. The worst case occurs in the 73th frame (3-D enhanced I frame) of Spiral Ball. It is found that there are two most-important ATM cell losses: one medium-important ATM cell loss and one least-important ATM cell loss in the transmission of the 3-D enhanced I frame. The medium- and least-important ATM 

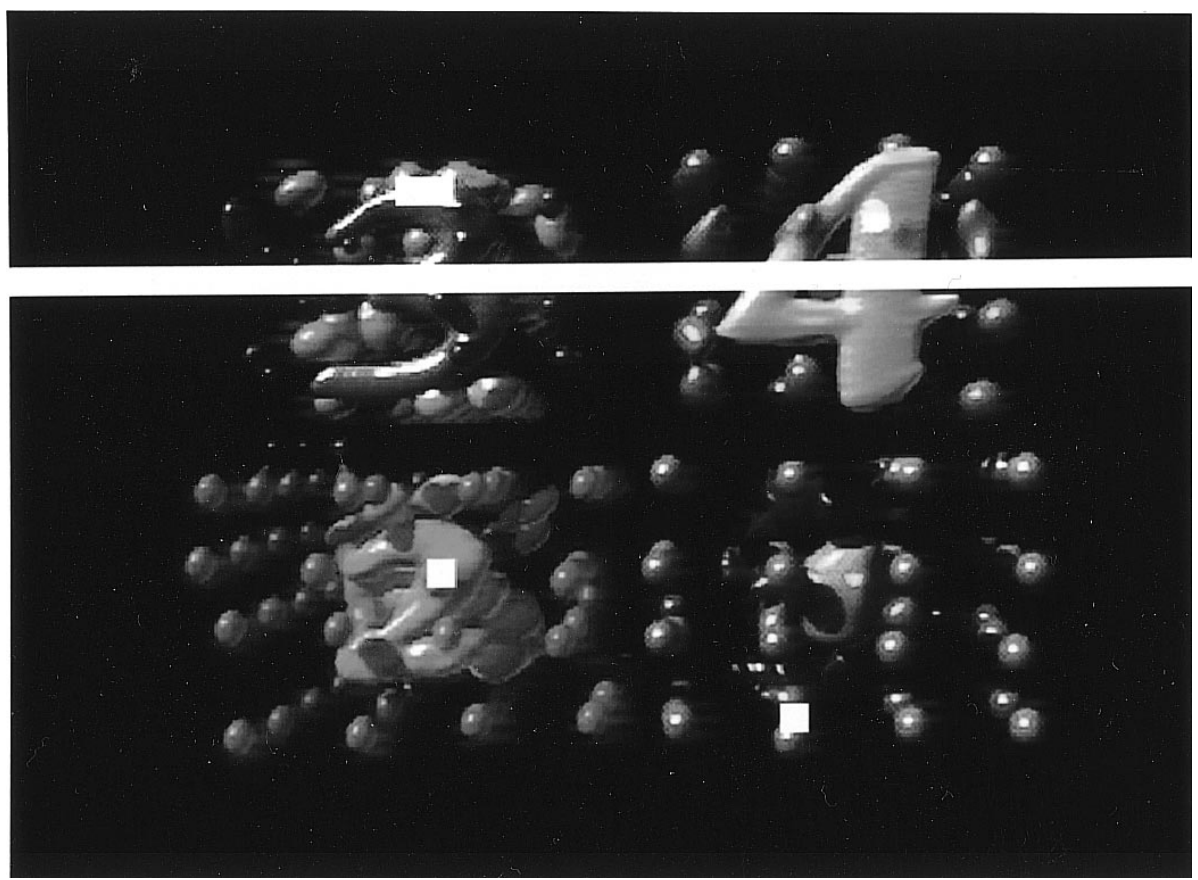

Fig. 14. Received I frame (frame 73) in left image due to four interfering two-way H.261 videos (white area denotes a lost area).

cells containing the second and third highest priority high-frequency detail subimages are visually less important than the most-important ATM cell containing the low-resolution subimages and have very little impact on video quality since over $80 \%$ of the image data are concentrated in the low-resolution subimages. Fig. 14 shows the received I frame in the left image with four lost areas, where the received I frame is a wavelet composition of the received pure low-resolution I frame which does not include the disparity information, the received second and third highest priority detail subimages. Note that the white area ( $Y=255, C_{b}=0, C_{r}=0$ ) represents a lost area since the background of each picture frame is black. Observing Fig. 14 from top to bottom, it is found that the second and fourth lost areas are caused by the loss of two most-important ATM cells, and the first and third lost areas are caused by the bit errors in the payload when their associated ATM cells are successful. Especially, the cell loss containing a slice header results in the loss of a slice of subsequent macroblocks in the second area. The fourth area is damaged when its associated lost cell contains the pure data belonging to the I frame generated from only the left low-resolution image. The first lost area is caused by a damaged macroblock header. The damage rates for dc and ac coefficients are found to be $0.0721 \%$ and $12.51 \%$, respectively. The third area is lost when both the dc and ac coefficients have been damaged simultaneously. Moreover, from Fig. 14, it is seen that the remaining area in the picture seems visually good; even with $12.51 \%$ of ac coefficients, both the medium- and least-important ATM cells have been damaged. On the other hand, Fig. 15 shows the received right image which is reconstructed from its corresponding left image (I-frame) by using the disparity-compensated method. The first, second, third, and fifth lost areas in the right image correspond to the first, second, third, and fourth lost areas belonging to the left image, respectively. The additional fourth lost area in the right image is caused by a damaged disparity vector in the payload. The damage rate for disparity-compensated prediction error is $15.21 \%$. Fig. 16 shows the recovered I frame in the left image by using the multiresolution multidirectional interpolation (spatial error concealment). The picture quality of Fig. 16 is improved at the performance of $\operatorname{PSNR}_{Y}=$ $32.48 \mathrm{~dB}, \mathrm{PSNR}_{U}=35.65 \mathrm{~dB}$, and $\mathrm{PSNR}_{V}=38.275 \mathrm{~dB}$. Fig. 17 illustrates the right image recovered from the previously concealed reference left image (I-frame) by using the disparity error concealment technique. The picture quality performance of Fig. 17 is $\operatorname{PSNR}_{Y}=30.84 \mathrm{~dB}, \mathrm{PSNR}_{U}=33.69 \mathrm{~dB}$, and $\mathrm{PSNR}_{V}=36.92 \mathrm{~dB}$.

It is found that the received $P$ frame (frame 76) in the left image has three lost areas, where the third lost area is caused by the loss of a most-important ATM cell containing a slice header and the other lost areas are caused by a damaged motion vector (first lost area) and a damaged macroblock header (second lost area) belonging to the payload when their associated ATM cells are successful, respectively [35]. Actually, there are two ATM cell losses in the received P frame. However, the other one is the medium-important ATM cell which has little impact on video quality. No damage occurs in the disparity vectors. Therefore, the lost areas in the right image are identical to that of the left image. The damage rates for both the motion- and disparity-compensated prediction errors are $8.68 \%$ and $9.91 \%$, respectively. The damaged $\mathrm{P}$ frame in the left image is recovered by using the iterative pattern matching method (temporal error concealment) with respect to the previously recovered reference I frame. Meanwhile, the damaged right image is recovered from the previously concealed $\mathrm{P}$ frame in the left image by using the disparity error concealment. Reference [35] has shown that the PSNR's of both the recovered images are $\mathrm{PSNR}_{Y}=29.56 \mathrm{~dB}$, $\mathrm{PSNR}_{U}=31.08 \mathrm{~dB}$, and $\mathrm{PSNR}_{V}=34.31 \mathrm{~dB}$ for the left 

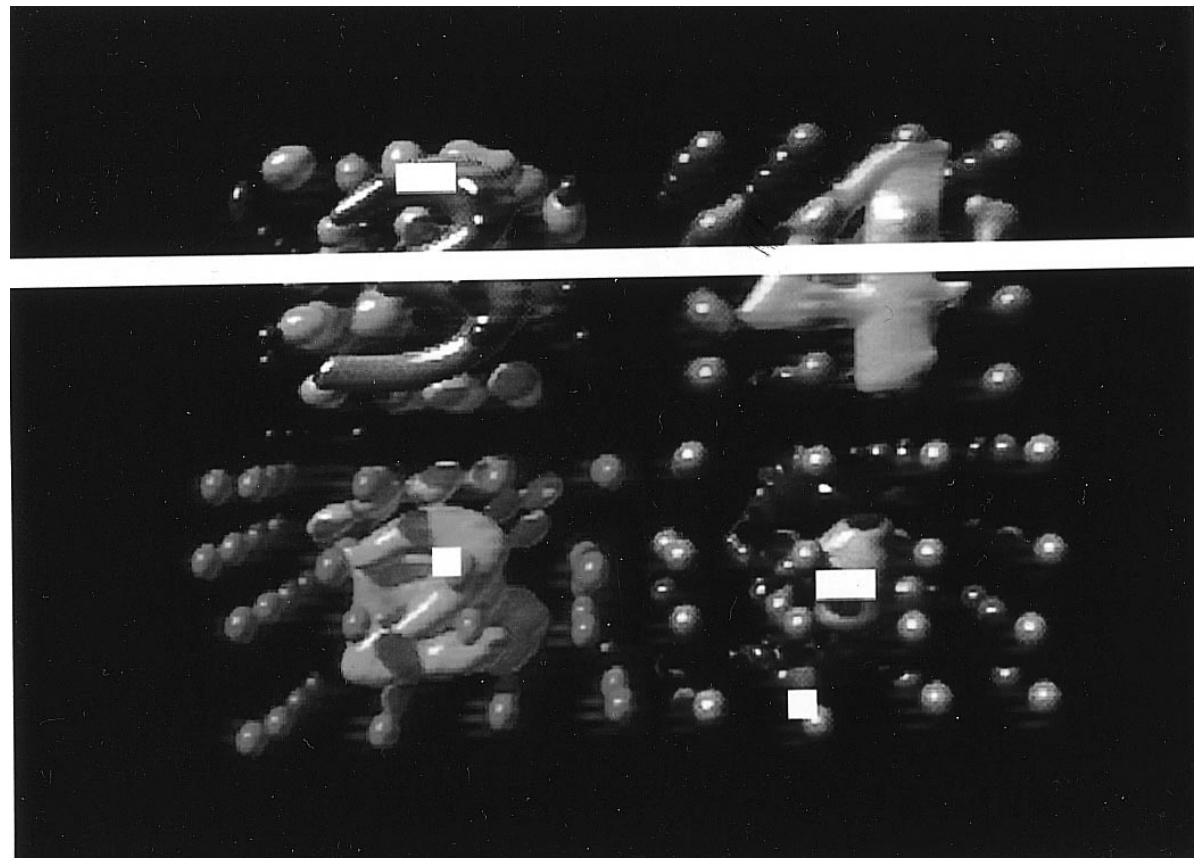

Fig. 15. Received right image (frame 73) due to four interfering two-way H.261 videos (white area denotes a lost area).

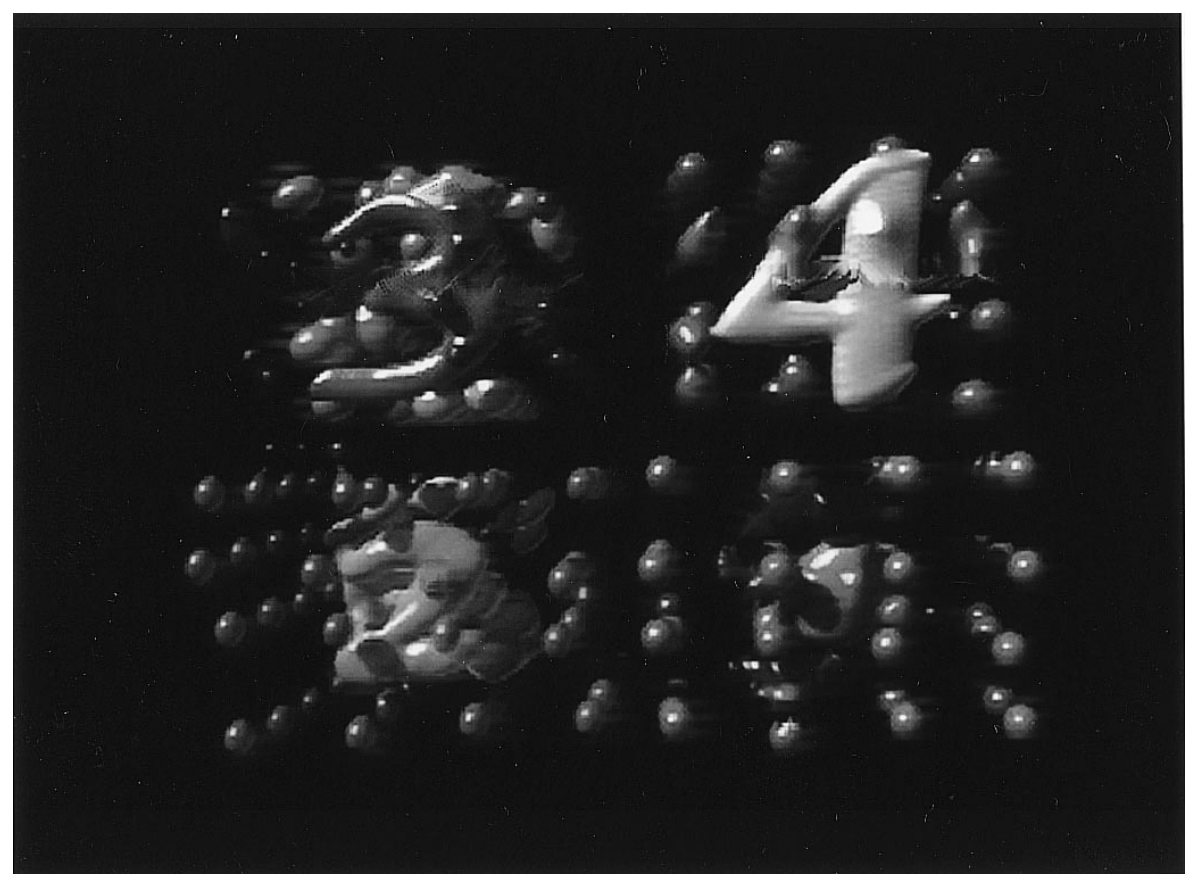

Fig. 16. Recovered I frame (frame 73) in left image by multiresolution multidirectional interpolation (spatial error concealment).

image and $\mathrm{PSNR}_{Y}=28.32 \mathrm{~dB}, \mathrm{PSNR}_{U}=29.76 \mathrm{~dB}$, and $\mathrm{PSNR}_{V}=32.51 \mathrm{~dB}$ for the right image. Moreover, [35] shows that the received B frame (frame 74) in the left image has two lost areas, where the second lost area is caused by the loss of a most-important ATM cell containing a slice header and the first lost area is caused by a damaged macroblock header belonging to the payload in a successful ATM cell. The additional lost area (first area) in the received right image is caused by a damaged disparity vector. The damage rates for both the motion- and disparity-compensated prediction errors are $5.41 \%$ and $6.78 \%$, respectively. The damaged $\mathrm{B}$ frames in the left image and its corresponding damaged right image are recovered by using the temporal and disparity error concealment techniques, respectively. The PSNR's of both the recovered images are PSNR $_{Y}=$ $27.52 \mathrm{~dB}, \mathrm{PSNR}_{U}=29.78 \mathrm{~dB}$, and PSNR $V=31.84 \mathrm{~dB}$ for the left image and $\mathrm{PSNR}_{Y}=26.8 \mathrm{~dB}, \mathrm{PSNR}_{U}=28.025 \mathrm{~dB}$, and $\mathrm{PSNR}_{V}=31.18 \mathrm{~dB}$ for the right image, respectively. Note that the damaged $B$ frame in the left image has been recovered from 


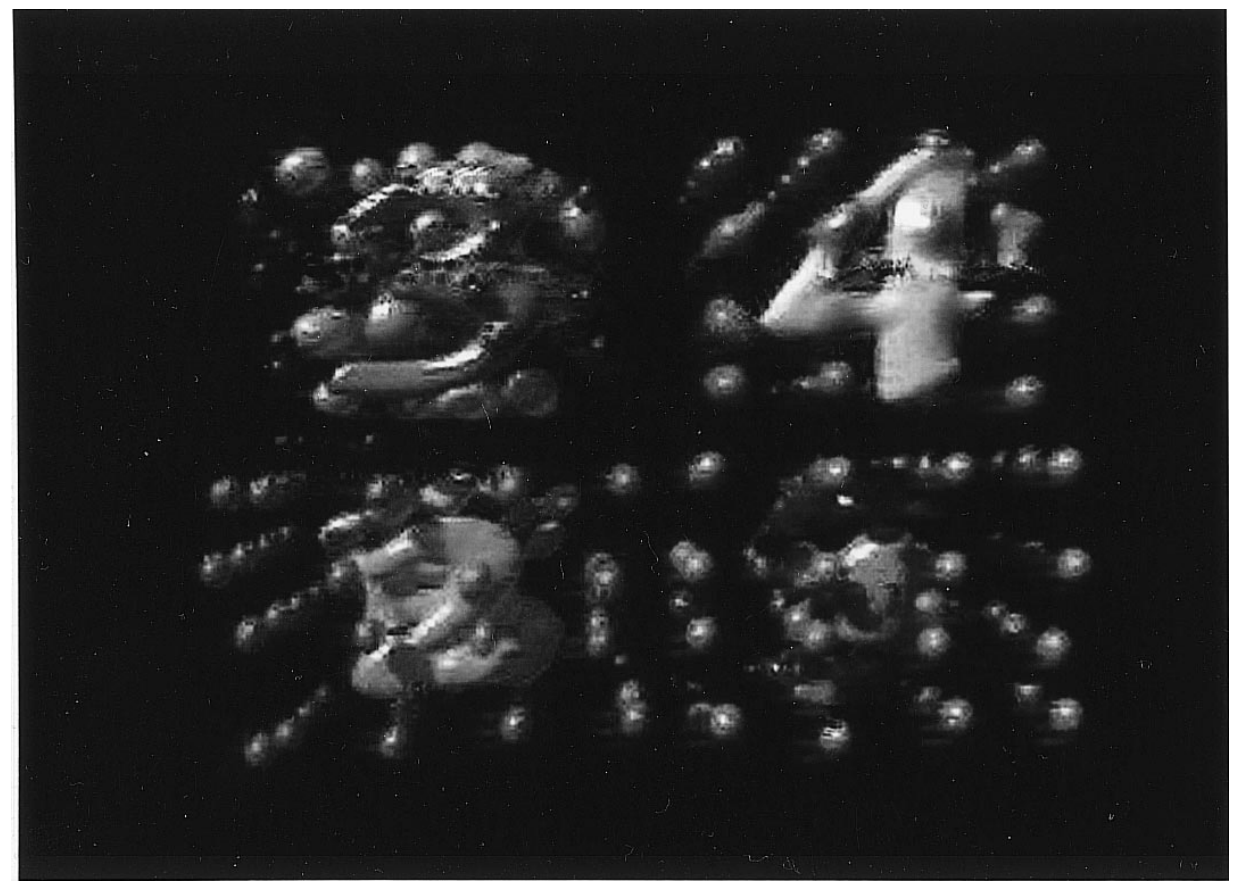

Fig. 17. Recovered right image (frame 73) by disparity error concealment based on iterative pattern matching method.

both the previously concealed I and P frames by using the temporal error concealment technique. The damaged right image has been recovered from the previously concealed B frame in the left image by using disparity error concealment technique.

\section{CONCLUSION}

An MPEG-based multiresolution 3-D video transmission over ATM-based multicode CDMA channels was investigated in this paper. An MPEG-based joint motion/disparity-compensated wavelet multiresolution coding based on mixed-resolution psychophysical experiments is proposed to achieve the high-compression ratio for typical 3-D stereoscopic image sequences, without any significant loss in the perceived 3-D image quality. The ATM cells have been generated according to both the statistical characteristics and the importance of data types in the 3-D video. A shortened $\mathrm{BCH}(31,16,3)$ code is used to effectively protect the ATM cell header in order to ensure the average number of ATM cell losses in both the left and right picture frames in the order of $\mathrm{O}(1)$. On the other hand, an adaptive FEC code combining scheme with unequal error protection is applied to the payload in accordance with its priority level in both spatial and temporal domains and the importance of its associated data type. Finally, the information loss in the damaged 3-D video can be concealed by using the spatial and temporal error concealment techniques for the damaged left image and by using the disparity error concealment technique for the damaged right image. Moreover, it can be shown that our system with a little modification is particularly suitable for Sarnoff's MPEG-4 video codec [39] which is actually a combination of wavelet-based multiresolution coding and motion-compensated coding. In the near future, our work is targeted at improving the system performance and reducing the BER floor further by concatenating the inner turbo code with the outer $\mathrm{BCH}$-based code combining via the wide CDMA (WCDMA) system testbed.

\section{REFERENCES}

[1] M. G. Perkins, "Data compression of stereopairs," IEEE Trans. Commun., vol. 40, pp. 684-696, Apr. 1992.

[2] A. Tamataoui and C. Labit, "Constrained disparity and motion estimation for 3-DTV image sequence coding," Signal Processing: Image Communication, vol. 4, pp. 45-54, Nov. 1991.

[3] D. Tzovaras et al., "Evaluation of multiresolution block matching techniques for motion and disparity estimation," Signal Processing: Image Communication, vol. 6, pp. 59-67, 1994.

[4] S. Lethurman et al., "A multiresolution framework for stereoscopic image sequence compression," in Proc. 1st IEEE Int. Conf. Image Processing, Austin, TX, Nov. 1994, pp. 361-365.

[5] Y. Q. Zhang and S. Zafar, "Motion-compensated wavelet transform coding for color video compression," IEEE Trans. Circuits Syst. Video Technol., vol. 2, pp. 285-296, Sept. 1992.

[6] S. Mallt, "A theory for multiresolution signal decomposition: The wavelet representation," IEEE Trans. Pattern Anal. Mach. Intell., vol. 11, pp. 647-693, July 1989.

[7] A. Grossmann and J. Morlet, "Decomposition of Hardy functions into square integrable wavelets of constant shape," SIAM J. Math., vol. 15, pp. 723-736, 1984.

[8] D. Raychaudhuri and N. Wilson, "ATM based transport architecture for multiservices wireless personal communication network," IEEE J. Select. Commun., pp. 1401-1414, Oct. 1994.

[9] D. LeGall, "MPEG: A video compression standard for multimedia applications," Comm. ACM, vol. 34, no. 4, pp. 46-58, 1991.

[10] D. Moore and M. Rice, "Variable rate error control for wireless ATM network," in Proc. ICC '95, pp. 988-992.

[11] M. J. Mctiffin, "Mobile access to an ATM network using a CDMA air interface," IEEE J. Select. Areas Commun., vol. 12, pp. 900-908, June 1994.

[12] J. B. Cain and D. N. McGregor, "A recommended error control architecture for ATM networks with wireless links," IEEE Select. Areas Commun., vol. 15, pp. 16-28, Jan. 1997.

[13] J. Zhang, M. R. Frater, J. F. Amold, and T. M. Percival, "MPEG2 video services for wireless ATM networks," IEEE Select. Areas Commun., vol. 15, pp. 119-128, Jan. 1997.

[14] C. L. I et al., "Performance of multi-code CDMA wireless personal communication networks," in 1995 IEEE 45th Veh. Technol. Conf., Chicago, IL, pp. 907-911. 
[15] M. H. Fong, V. K. Bhargava, and Q. Wang, "Concatenated orthogonal/PN spreading sequences and their application to cellular DS-CDMA systems with integrated traffic," IEEE J. Select. Areas Commun., vol. 14, pp. 547-557, Apr. 1996.

[16] P. R. Chang, "Spreading spectrum CDMA system for subband image transmission," IEEE Trans. Veh. Technol., vol. 46, pp. 80-95, Feb. 1997.

[17] Y. Q. Zhang and X. Lee, "Performance of MPEG codes in the presence of errors," J. Visual Commun. Image Representation, vol. 5, pp. 379-387, Dec. 1994.

[18] Y. Wang, Q. F. Zhu, and L. Shaw, "Maximally smooth image recovery in transform coding," IEEE Trans. Commun., vol. 41, pp. 1544-1551, Oct. 1993.

[19] I. W. Tsai and C. L. Huang, "Hybrid cell loss concealment methods for MPEG-II-based packet video," Signal Processing: Image Communication, vol. 9, pp. 99-124, 1997.

[20] W. Kwok and H. Sun, "Multi-directional interpolation for spatial error concealment," IEEE Trans. Consumer Electron., vol. 39, pp. 455-460, Aug. 1993.

[21] X. Lee, Y. Q. Zhang, and A. Leon-Garcia, "Information loss recovery for block-based image coding techniques-A fuzzy logic approach," IEEE Trans. Image Processing, vol. 4, pp. 259-273, Mar. 1995.

[22] S. Aign and K. Fazel, "Error detection and concealment measures in MPEG-2 video decoder," in Proc. Int. Workshop HDTV '94, Torino, Italy, Oct. 1994.

[23] A. Narula and J. S. Lim, "Error concealment techniques for an all-digital high-definition television system," SPIE, vol. 2094, 1993.

[24] S. M. Lei, "Forward error correction codes for MPEG2 over ATM," IEEE Trans. Circuits Syst. Video Technol., vol. 4, pp. 200-203, Apr. 1994

[25] G. C. Clark, Jr., Error-Correction Coding for Digital Communications. New York: Plenum, 1981.

[26] V. Parthasarathy, J. W. Modestino, and K. S. Vastola, "Design of transport coding scheme for high-quality video over ATM networks," IEEE Trans. Circuits Syst. Video Technol., vol. 7, pp. 358-376, Apr. 1997.

[27] J. G. Proakis, Digital Communications. New York: McGraw-Hill, 1983

[28] H. Ohta and T. Kitami, "A cell loss recovery method using FEC in ATM networks," IEEE Select. Areas Commun., vol. 9, pp. 1471-1483, Dec. 1991.

[29] H. Liu and M. El Zarki, "Transmission of video telephony images over wireless channels," Wireless Networks, vol. 2, pp. 219-228, 1996.

[30] M. Kavehrad and B. Ramamurthi, "Direct-sequence spread spectrum with DPSK modulation and diversity for indoor wireless communications," IEEE Trans. Commun., vol. COM-35, pp. 224-236, Feb. 1987.

[31] M. K. Ozkan, M. I. Sezan, and A. M. Tekalp, "Adaptive motion-compensation filtering of noisy image sequences," IEEE Trans. Circuits Syst. Video Technol., vol. 3, pp. 277-290, Aug. 1993.

[32] M. Kavehrad and B. Ramamurthi, "Direct-sequence spread spectrum with DPSK modulation and diversity for indoor wireless communications," IEEE Trans. Commun., vol. COM-35, pp. 224-236, Feb. 1987.

[33] M. K. Ozkan, M. I. Sezan, and A. M. Tekalp, "Adaptive motion-compensation filtering of noisy image sequences," IEEE Trans. Circuits Syst. Video Technol., vol. 3, pp. 277-290, Aug. 1993.

[34] M. B. Pursley, "Spread spectrum multiple access communications," in Multi-User Communication Systems. New York: Springer-Verlag, 1981, pp. 139-189.

[35] P. R. Chang, "Error concealment techniques for 3-D video via wireless CDMA network,", to be published.

[36] J. D. Andersen, "Turbo codes extended with outer BCH code," Electron. Lett., vol. 32, pp. 2059-2060, Oct. 1996.
[37] P. R. Chang and M. F. Wu, "A wavelet multiresolution compressikon technique for 3-D stereoscopic image sequence based on mixed-resolution psychophisical experiments,", to be published.

[38] P. R. Chang and C. F. Lin, "Wireless ATM-based multi-code CDMA transport architecture for MPEG2 video transmission," Proc. IEEE, vol. 87, pp. 1807-1824, Oct. 1999.

[39] Y. Q. Zhang et al., "A zerotree wavelet video coder," Special Issue on MPEG-4, IEEE Trans. Circuits Syst. Video Technol., vol. 7, pp. 109-118, Feb. 1997.

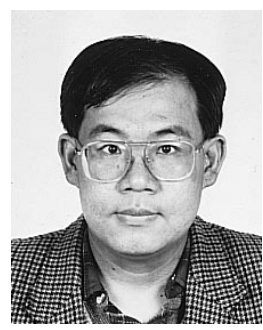

Po-Rong Chang (M'87) received the B.S. degree in electrical engineering from the National Tsing-Hua University, Hsinchu, Taiwan, R.O.C., in 1980, the M.S. degree in communication engineering from National Chiao-Tung University, Hsinchu, in 1982, and the Ph.D. degree in electrical engineering from Purdue University, West Lafayette, IN, in 1988, respectively.

From 1982 to 1984, he was a Lecturer at the Chinese Air Force Telecommunication and Electronics School for his two-year military service. From 1984 to 1985 , he was an Instructor of Electrical Engineering at the National Taiwan Institute of Technology, Taipei, Taiwan. From 1989 to 1990, he was a Project Leader in charge of the SPARC chip design team at ERSO, Industrial Technology and Research Institute, Chu-Tung, Taiwan. Currently, he is a Professor of Communication Engineering at National Chiao-Tung University. His current interests include wide-band CDMA systems, wireless multimedia communications, fuzzy neural networks, and low-power design for wireless communications.

Dr. Chang was the recipient of the Best Paper Award on the quality control for semiconductor manufacturing in 1990, Taiwan.

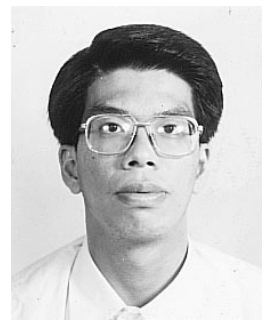

Chin-Feng Lin was born in Taiwan, R.O.C., in 1965. He received the B.S. degree in electrical engineering from Chung-Yung University, Taiwan, in 1996 and the M.S. degree in electrical engineering from Chung-Hua University, Taiwan, in 1998, respectively. Currently, he is working towards the $\mathrm{Ph} . \mathrm{D}$. degree in communication engineering at the National Chiao-Tung University, Hsinchu, Taiwan.

His research interests include CDMA systems and wireless multimedia communications.

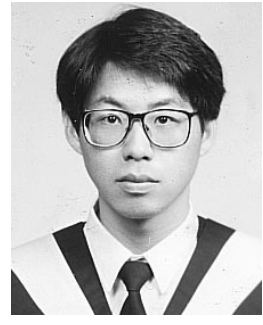

M. J. Wu received the B.S. degree in physics from National Taiwan University, Taipei, Taiwan, R.O.C. $\mathrm{He}$ is currently working towards the M.S. degree in communication engineering at the National Chiao-Tung University, Hsinchu, Taiwan. 